\title{
A neural mechanism for detecting object motion during self-motion
}

\author{
HyungGoo R. Kim ${ }^{1,2,4}$, Dora E. Angelaki ${ }^{3}$, and Gregory C. DeAngelis ${ }^{4}$
}

${ }^{1}$ Center for Neuroscience Imaging Research, Institute for Basic Science, Suwon 16419, Republic of Korea

${ }^{2}$ Department of Biomedical Engineering, Sungkyunkwan University, Suwon 16419, Republic of Korea

${ }^{3}$ Center for Neural Science, New York University, New York, New York, USA

${ }^{4}$ Department of Brain and Cognitive Sciences, Center for Visual Science, University of Rochester, Rochester, New York USA

Acknowledgements:

We thank Johnny Wen for programming assistance, as well as Swati Shimpi, Emily Murphy, and Dina Graf for assistance with training animals. This work was supported by NEI R01 grant EY013644, NINDS U19 grant NS118246, and by an NEI Core grant (EY001319). 


\section{ABSTRACT}

Detecting objects that move in a scene is a fundamental computation performed by the visual system. This computation is greatly complicated by observer motion, which causes most objects to move across the retinal image. How the visual system detects scene-relative object motion during self-motion is poorly understood. Human behavioral studies suggest that the visual system may identify local conflicts between motion parallax and binocular disparity cues to depth, and may use these signals to detect moving objects. We describe a novel mechanism for performing this computation based on neurons in macaque area MT with incongruent depth tuning for binocular disparity and motion parallax cues. Neurons with incongruent tuning respond selectively to scene-relative object motion and their responses are predictive of perceptual decisions when animals are trained to detect a moving object during selfmotion. This finding establishes a novel functional role for neurons with incongruent tuning for multiple depth cues.

\section{INTRODUCTION}

When an observer moves through the environment, image motion on the retina generally includes components caused by self-motion and objects that move relative to the scene, both of which depend on the depth structure of the scene. Because selfmotion typically causes a complex pattern of image motion across the visual field (optic flow, Gibson et al. 1959; Koenderink and van Doorn 1987), detecting the movement of objects relative to the world can be a difficult task for the brain to solve. An object that is moving in the world might appear to move faster or slower in the image than objects that are stationary in the scene, depending on the specific viewing geometry. Thus, a critical computational challenge for detecting scene-relative object motion is to identify components of image motion that are not caused by one's self-motion and the static depth structure of the scene. This is a form of causal inference problem (French and DeAngelis 2020; Shams and Beierholm 2010).

Object movement may be relatively easy to distinguish from self-motion when the object's temporal motion profile is clearly different from that of image motion resulting from self-motion (Layton and Fajen 2016b) or when the object moves with a direction 
that is incompatible with self-motion (Royden and Connors 2010). Neural mechanisms with center-surround interactions in velocity space have been proposed as potential solutions to the problem of detecting object motion under these types of conditions (Royden and Holloway 2014; Royden et al. 2015). However, the brain has a remarkable ability to detect object motion even under conditions in which the image velocity of a moving object is very similar to that of stationary background elements during selfmotion. Rushton et al (2007) demonstrated that object movement relative to the scene "pops out" when 3D structure is specified by binocular disparity cues, but not in the absence of disparity cues. They suggested that disparity cues help the visual system to discount the global flow field resulting from self-motion, thereby identifying object motion. How the brain might achieve this computation has remained a mystery.

We previously reported that many neurons in area MT have incongruent tuning for depth defined by binocular disparity and motion parallax cues (Nadler et al. 2013). We speculated that such neurons might play a role in detecting object motion during self-motion by responding selectively to local conflicts between disparity and motion parallax cues (Kim et al. 2016a; Nadler et al. 2013). Here, we test this hypothesis directly by recording from MT neurons while monkeys perform a task that requires detecting object motion during self-motion. We show that monkeys perform this task based mainly on local differences in depth as cued by disparity and motion parallax. We demonstrate that MT neurons with incongruent tuning for depth based on disparity and motion parallax are generally more sensitive to scene-relative object motion, and that their responses correlate preferentially with animals' perceptual decisions. We further demonstrate that training a linear decoder to detect object motion based on MT responses largely reproduces our major empirical result. Our findings establish a novel neural mechanism for detecting moving objects during self-motion, thus revealing a sensory substrate for a specific form of causal inference. Because this mechanism relies on sensitivity to local discrepancies between disparity and motion parallax cues, it allows detection of object motion without the need for more complex computations that discount the global flow field. Thus, this mechanism for detecting object motion in the world may be relatively economical for the nervous system to implement. 


\section{METHODS}

\section{Subjects and surgery}

Two male monkeys (macaca mulatta, 8-12 kg) participated in these experiments. Standard aseptic surgical procedures under gas anesthesia were performed to implant a head restraint device. A Delrin (Dupont) ring was attached to the skull using a combination of dental acrylic, bone screws, and titanium inverted T-bolts (see Gu et al. 2006 for details). To monitor eye movements using the magnetic search coil technique, a scleral coil was implanted under the conjunctiva of one eye.

A recording grid made of Delrin was affixed inside the ring using dental acrylic. The grid $(2 \times 4 \times 0.5 \mathrm{~cm})$ contains a dense array of holes spaced $0.8 \mathrm{~mm}$ apart. Under anesthesia and using sterile technique, small burr holes $(\sim 0.5 \mathrm{~mm}$ diameter) were drilled vertically through the recording grid to allow the penetration of microelectrodes into the brain via a transdural guide tube. All surgical procedures and experimental protocols were approved by University Committee on Animal Resources at the University of Rochester.

\section{Experimental apparatus}

In each experimental session, animals were seated in a custom-built primate chair that was secured to a six degree-of-freedom motion platform (MOOG 6DOF2000E). The motion platform was used to generate passive body translation along an axis in the fronto-parallel plane and the trajectory of the platform was controlled in real time at $60 \mathrm{~Hz}$ over a dedicated Ethernet link (see Gu et al. 2006 for details). A field coil frame ( $\mathrm{C}-\mathrm{N}-\mathrm{C}$ Engineering) was mounted on top of the motion platform to measure eye movements.

Visual stimuli were rear-projected onto a $60 \times 60 \mathrm{~cm}$ tangent screen using a stereoscopic projector (Christie Digital Mirage S+3K) which was also mounted on the motion platform (Gu et al. 2006). The display screen was attached to the front side of the field coil frame. To restrict the animal's field of view to visual stimuli displayed on the tangent screen, the sides and top of the field coil frame were covered with matte black enclosures. Viewed from a distance of $\sim 30 \mathrm{~cm}$, the display subtended $\sim 90^{\circ} \times 90^{\circ}$ of visual angle. 
To generate accurate visual simulations of the animal's movement through a virtual environment, an OpenGL camera was placed at the location of one eye and the camera moved precisely according to the movement trajectory of the platform. Since the motion platform has its own dynamics, we characterized the transfer function of the motion platform, as described previously (Gu et al. 2006), and we generated visual stimuli according to the predicted motion of the platform. To account for a delay between the command signal and the actual movement of the platform, we adjusted a delay parameter to synchronize visual motion with platform movement. Synchronization was confirmed by presenting a world-fixed target in the virtual environment and superimposing a small spot by a room-mounted laser pointer while the platform is in motion (Gu et al. 2006).

\section{Electrophysiological recordings}

We recorded extracellular single unit activity using single-contact tungsten microelectrodes (FHC Inc.) having a typical impedance of 1-3 M $\Omega$. The electrode was loaded into a transdural guide tube and was manipulated with a hydraulic micromanipulator (Narishige). The voltage signal was amplified and filtered $(1 \mathrm{kHz}-6 \mathrm{kHz})$ using conventional hardware (BAK Electronics). Single unit spikes were detected using a window discriminator (BAK Electronics), whose output was time-stamped with $1 \mathrm{~ms}$ resolution.

Eye position signals were digitized at $1 \mathrm{kHz}$, then digitally filtered and down sampled to $200 \mathrm{~Hz}$ (TEMPO, Reflective Computing). The raw voltage signal from the microelectrode was digitized and recorded to disk at $25 \mathrm{kHz}$ using a Power1401 data acquisition system (Cambridge Electronic Design). If necessary, single units were resorted off-line using a template-based method (Spike2, Cambridge Electronic Design).

The location of area MT was initially identified in each animal through analysis of structural MRI scans, which were segmented, flattened, and registered with a standard macaque atlas using CARET software (Van Essen et al. 2001). The position of area MT in the posterior bank of the superior temporal sulcus (STS) was then projected onto the horizontal plane, and grid holes around the projection area were explored systematically in mapping experiments. In addition to the MRI scans, the physiological properties of 
neurons and the patterns of gray matter and white matter encountered along electrode penetrations provided essential evidence for identifying MT. In a typical electrode penetration through the STS that encounters area MT, we first encounter neurons with large receptive fields and visual motion sensitivity (as expected for area MSTd). This is typically followed by a very quiet region as the electrode passes through the lumen of the STS, and then area MT is the next region of gray matter. As expected from previous studies, receptive fields of MT neurons are much smaller than those in MSTd (Komatsu and Wurtz 1988) and some MT neurons exhibit strong surround suppression (DeAngelis and Uka 2003) which is typically not seen in MSTd. Confirming a putative localization of the electrode to MT, we observed gradual changes in the preferred direction, preferred disparity, and receptive field location of multiunit activity, consistent with those described previously (Albright et al. 1984; DeAngelis and Newsome 1999).

\section{Visual stimuli}

Visual stimuli were generated by a custom-written $\mathrm{C}++$ program using the OpenGL 3D graphics library, and were displayed using a hardware-accelerated OpenGL graphics card (NVIDIA Quadro FX 1700). The location of the OpenGL camera was matched to the location of the animal's eye, and images were generated using perspective projection. We calibrated the display such that the virtual environment had the same spatial scale as the physical space through which the platform moved the animal. To view stimuli stereoscopically, animals wore anaglyphic glasses with red and green filters (Kodak Wratten 2 Nos. 29 and 61, respectively). The crosstalk between eyes was measured using a photometer and found to be very small $(0.3 \%$ for the green filter and $0.1 \%$ for the red filter).

Stimulus to measure depth tuning from motion parallax. We used an established procedure to generate random-dot stimuli to measure depth tuning from motion parallax (Nadler et al. 2008). A circular aperture having slightly greater ( 10\%) diameter than optimal size was located over the center of the receptive field of the neuron under study. The position of each dot in the image plane was generated by independently choosing random horizontal and vertical locations within the aperture. To present stimuli such that they appear to lie in depth at a specific equivalent disparity, the set of random dots 
within the circular aperture was ray-traced onto a cylinder corresponding to the desired equivalent disparity, as described in detail previously (Nadler et al. 2008). This raytracing procedure ensured that the size, location, and density of the random dot patch were constant across simulated depths. Size and occlusion cues were eliminated by rendering transparent dots with a constant retinal size (0.39 deg). Critically, this procedure removed pictorial depth cues and rendered the visual stimulus depth-sign ambiguous, thus requiring interaction of retinal object motion with either extra-retinal signals (Nadler et al. 2009) or global visual motion cues (Kim et al. 2015b) that specify eye rotation relative to the scene.

The above description assumes lateral translation of the observer in the horizontal plane. However, in our experiments, animals were translated along an axis in the fronto-parallel plane that was aligned with the preferred-null axis of the neuron under study (to elicit robust neural responses). In this case, we rotated the virtual stimulus cylinder about the naso-occipital axis such that the axis of translation of the observer was always orthogonal to the long axis of the cylinder. This ensures that dots having the same equivalent disparities produce the same retinal speeds regardless of the axis of observer translation (Nadler et al. 2008).

Stimulus for object detection task. Visual stimuli for the main task consisted of a dynamic target object (which could be either moving or stationary in the world), one or three stationary objects (distractors), and a cloud of background dots that appeared outside of a central masked region (Supplementary Fig. 1A, Supplementary Video 1). Background dots were masked out of this central region around the target and distractor objects to avoid having the background dots directly stimulate the receptive field of the neuron under study. The two-object version of the task (one dynamic target, one stationary distractor) was used in all neural recording experiments, whereas the fourobject task (one dynamic target, 3 stationary distractors) was used during training and in some behavioral control experiments.

For the two-object task, one object was located in the center of the receptive field of the neuron under study, and the other object was presented on the opposite side of the fixation target (180 deg apart) at the same eccentricity (Fig. 1A, B). For the fourobject task, one object was centered on the receptive field, and the other three objects 
were distributed equally (90 deg apart) around the fixation target at equal eccentricities. To present each object at the same retinal position regardless of its depth, the positions of objects were initially determined in screen coordinates and then were ray-traced onto surfaces in the simulated environment (Supplementary Fig. 1B, left).

Each object was rendered as a square-shaped "plate" of random dots (density: $1.1 \mathrm{dots} / \mathrm{deg}^{2}$ ), and was displayed binocularly as a red-green anaglyph. The retinal size of dots was constant ( $0.15 \mathrm{deg})$ regardless of object depth, such that dot size was not a depth cue. The target and distractor objects were all of the same retinal size (which was tailored to the receptive field of the neuron under study) regardless of their location in depth, such that the image size of objects was also not a depth cue. Thus, the only reliable cues to object depth were binocular disparity and motion parallax.

Dynamic target objects had two independent depth parameters, one based on binocular disparity $\left(\mathrm{d}_{\mathrm{BD}}\right)$ and the other based on motion parallax ( $\left.\mathrm{d}_{\mathrm{MP}}\right)$. The left-eye and right-eye half-images of the dynamic object were rendered based on the depth defined by binocular disparity, dBD. We then computed the image motion of the dynamic object during translation of the monkey such that it had motion parallax that was consistent with a different depth, dMP. Based on the predicted trajectory of the camera on each video frame, we ray-traced the position of the dynamic object (at dMP) onto the depth plane defined by binocular disparity, $\mathrm{d}_{\mathrm{BD}}$ (Supplementary Fig. 1B, right). This procedure ensures that the dynamic object had a particular difference in depth $(\Delta$ Depth, in equivalent disparity units) specified by (dMP - $\left.d_{B D}\right)$, but that it was not possible to detect the dynamic object solely based on its relative motion in the scene (Supplementary Fig. 2). In other words, when viewed monocularly, the image motion of the dynamic object would be consistent with that of a stationary object at dMP. When viewed binocularly, if $\Delta$ Depth $\neq 0$, the dynamic object's image motion would not be consistent with its depth specified by disparity, dBD.

\section{Experimental Protocol}

Preliminary measurements. After isolating the action potential of a single neuron, the receptive field was explored manually using a small (typically 2-3 deg) patch of random dots. The direction, speed, position, and binocular disparity of the random-dot 
patch were manipulated using a computer mouse, and instantaneous firing rates were plotted on a display interface that represents the spatial location of the patch in visual space and the stimulus velocity in a direction-speed space. This procedure was used to estimate the location and size of the receptive field as well as to estimate the neuron's preferences for direction, speed, and binocular disparity.

After these qualitative tests, we measured the direction, speed, binocular disparity, and size tuning of each neuron using quantitative protocols (DeAngelis and Uka 2003). Each of these measurements was performed in a separate block of trials, and each distinct stimulus was repeated 3-5 times. Direction tuning was measured with random dots that moved in eight different directions separated by 45 deg. Speed tuning was measured, at the preferred direction, with random dot stimuli that moved at speeds of $0,0.5,1,2,4,8,16$, and $32 \mathrm{deg} / \mathrm{s}$. The stimuli in our main task contained speeds of motion that were $<7 \mathrm{deg} / \mathrm{s}$. If a neuron gave very little response $(<5 \mathrm{spk} / \mathrm{s})$ to these slow speeds, the neuron was not studied further. Next, the spatial profile of the receptive field was measured by presenting a patch of random dots at all locations on a $4 \times 4$ grid that covered the receptive field. The height and width of the grid were 1.5-2.5 times larger than the estimated receptive field size, and each small patch was approximately $1 / 4$ the size of the receptive field. Responses were fitted by a 2D Gaussian function to estimate the center location and size of the receptive field. To measure binocular disparity tuning, a random dot stereogram was presented at binocular disparities ranging from -2 deg to +2 deg in steps of 0.5 deg. For this disparity tuning measurement, dots moved in the neuron's preferred direction and speed. Finally, size tuning was measured with random-dot patches having diameters of $0.5,1,2,4,8,16$, 32 deg.

Depth tuning from motion parallax was then measured as described previously (Nadler et al. 2008; Nadler et al. 2013). Dots were presented monocularly and were rendered at one of nine simulated depths based on their motion ( -2 deg to +2 deg of equivalent disparity in steps of $0.5 \mathrm{deg}$ ), in addition to the null condition in which only the fixation target was presented. Each distinct stimulus was repeated 6-10 times. During measurement of depth tuning from motion parallax, animals underwent passive whole-body translation which followed a modified sinusoidal trajectory along an axis in 
the frontoparallel plane (Supplementary Fig. 1C). To smooth the onset and offset, the $2 \mathrm{~s}$ sinusoidal trajectory was multiplied by a Gaussian function that was exponentiated to a large power as follows:

$$
G(t)=e^{-\frac{\left(t-t_{0}\right)^{n}}{\sigma^{n}}}
$$

where $t_{0}=1.0 \mathrm{~s}, \sigma=0.92$, and $n=22$. On half of the trials, platform movement started toward the neuron's preferred direction. On the other half, motion started toward the neuron's null direction (Supplementary Fig. 1C). During body translation, animals were required to maintain fixation on a world-fixed target, which required a compensatory smooth eye movement in the direction opposite to head movement.

Moving object detection task. We presented one dynamic (i.e., moving) object and one (or three) stationary object(s) while the animal experienced the modified sinusoidal lateral motion as described above. The animal was trained to identify the dynamic object by making a saccadic eye movement to it (Fig. 1A). At the beginning of each trial, the fixation target first appeared at the center of the screen. After the animal established fixation for $0.2 \mathrm{~s}$, the dynamic object, stationary object(s), and background cloud of dots appeared and began to move as the animal was translated sinusoidally for 2.1s (see Supplementary Video 1). Because the fixation target was world-fixed, translation of the animal required a counter-active smooth eye movement to maintain visual fixation. An electronic window around the fixation target was used to monitor and enforce pursuit accuracy. The initial size of the target window was 3-4 deg, and it shrunk to 2.1-2.8 deg after $250 \mathrm{~ms}$ of translation. This allowed the animal a brief period of time to initiate pursuit and execute a catch-up saccade to arrive on target. At the end of visual stimulation, both the fixation target and the visual stimuli disappeared and a choice target (0.4 deg in diameter) appeared at the center location of each object. The animal then attempted to make a saccadic eye movement to the location of the dynamic object, and received a liquid reward (0.2-0.4 ml) for correct answers.

Based on the preliminary tests described above, we set the axis of translation within the frontoparallel plane to align with the preferred-null axis of the neuron under study. In the main detection task, we systematically varied the depth discrepancy ( $\Delta$ Depth) between disparity and motion parallax cues for the dynamic object, to 
manipulate task difficulty (Supplementary Fig. 1B). $\Delta$ Depth is defined as the difference between depths specified by motion parallax and binocular disparity cues, (dMP - $\left.d_{B D}\right)$. Different values of $\Delta$ Depth were applied to the dynamic object around a fixed "pedestal depth" (red line, Supplementary Fig. 1B). For the vast majority of recording sessions, the pedestal depth was fixed at $-0.45 \mathrm{deg}$ (103/106 sessions), although it deviated from this value slightly in a few early experiments. We elected to use a fixed pedestal depth such that all neurons were tested with the same stimulus values, thereby allowing for decoding analyses (described below). The pedestal depth was chosen as the average midpoint between the preferred depths obtained from tuning curves for disparity and motion parallax, based on data from a previous study (Nadler et al. 2013). We used the following $\Delta$ Depth values: $-1.53,-0.57,-0.21,0,0.21,0.57,1.53 \mathrm{deg}$. Stationary objects were presented at one of seven possible depths $(-1.6$ deg to +1.6 deg in steps of 0.4 deg). The vast majority of recording sessions were conducted using these 'standard' pedestal depth, $\Delta$ Depth, and stationary depth values (101/106 sessions). Thus, the maximum range of depths of dynamic objects $(-1.215$ to $+0.315 \mathrm{deg})$ was well within the range of depths for stationary objects, which ensured that the animals could not perform the task solely based on depth outliers (either in binocular disparity or motion parallax). The identity of each object (dynamic/stationary) and its depth values were chosen from the above ranges randomly on each trial._Each $\Delta$ Depth value of the dynamic object was repeated at least 14 times (mean: 35, sd: 9.6).

For 3 sessions, a monkey performed the object detection task without binocular disparity cues in a fraction of trials (Supplementary Fig. 2, monocular condition). In this control condition, the visual stimulus (except for the fixation point) was displayed to only one eye in $16 \%$ of trials, while the rest of the task structure remained the same. Monocular conditions were presented in a small percentage of trials in order not to frustrate the animal, given that performance was poor on these monocular trials.

\section{Animal training procedure}

Although the object detection task is conceptually simple, it required extensive behavioral training, involving a number of steps. Here, we outline the series of operant conditioning steps required to teach animals to perform the task. Following basic chair 
training and habituation to the laboratory, animals were trained to maintain visual fixation on a target during sinusoidal translation of the motion platform.

Once smooth eye movements tracked the fixation target with pursuit gains approaching 0.9 , we initially trained animals to detect a moving object without any selfmotion, such that any motion of an object on the display resulted from object motion relative to the scene. After fixation, four objects appeared on the display and only one of them moved sinusoidally along a horizontal trajectory for $2.1 \mathrm{~s}$. In the early stages of this training, a saccade target appeared only at the location of the moving object.

Subsequently, we introduced a fraction of trials in which saccade targets appeared at the locations of all four objects, and we gradually increased the proportion of these trials. During this phase of training, the depths of the objects, as defined solely by binocular disparity since there was no self-motion, were randomly drawn from a uniform distribution spanning the range from -1.6 to $+1.6 \mathrm{deg}$, to help animals generalize the task.

Once animals performed the task well in the absence of self-motion, we began to introduce small amounts of sinusoidal self-motion, which induced subtle retinal image motion of all objects. During the initial stages of this training period, the dynamic object had a large motion amplitude such that it was quite salient relative to the motion of stationary objects that was due to self-motion. As the animals became accustomed to performing the task during self-motion, we gradually increased the magnitude of selfmotion (up to $2.8 \mathrm{~cm}$ ) and decreased the motion amplitude of the dynamic object. Once the retinal motion amplitude of the dynamic object became comparable to that of stationary objects, we began to introduce a depth discrepancy between disparity and motion parallax ( $\Delta$ Depth). That is, the motion trajectory of the dynamic object began to follow that of an object at a different depth, $d_{M P}$ (Supplementary Fig. 1B, right). We used a staircase procedure to train animals over a range of values of $\Delta$ Depth. During this phase of training, we interleaved three different pedestal depths (-0.51 deg, 0 deg, 0.51 deg) to help animals generalize the task, and we randomly chose the depths of the three stationary objects from the range -1.6 to $+1.6 \mathrm{deg}$.

Once we observed stable 'v-shaped' psychometric functions for all three pedestal depths over a span of more than 10 days (e.g., Supplementary Fig. 3A,B), we 
transitioned to the final stimulus configuration for recording experiments. To keep the number of stimulus conditions manageable for recording, this configuration included one pedestal depth and two objects (one dynamic, one stationary). Following recording experiments, we revisited the more general version of the task involving four objects and 3 pedestal depths to make sure that behavioral performance did not reflect any change in strategy (e.g., Supplementary Fig. 3C).

\section{Data analyses}

Regression analysis of behavior. We used multinomial regression to assess the relative contributions of $\mathrm{d}_{\mathrm{BD}}, \mathrm{d}_{\mathrm{MP}}$, and $\Delta$ Depth to perceptual decisions. If animals perform the task primarily based on the discrepancy between disparity and motion parallax cues to depth, we expect to see a much greater contribution of $\Delta$ Depth relative to $\mathrm{d}_{\mathrm{BD}}$ and $\mathrm{d}_{\mathrm{MP}}$. For each possible choice location, i, (i.e., a chosen location or a notchosen location), we performed the following regression:

$$
\log \left(\frac{P\left(\text { choice }_{i}\right)}{1-P\left(\text { choice }_{i}\right)}\right)=\beta_{0}+\sum_{j}^{N}\left(\beta_{B D, i, j}\left|d_{B D, i, j}\right|+\beta_{M P, i, j}\left|d_{M P, i, j}\right|+\beta_{\Delta, \mathrm{i}, \mathrm{j}}\left|\Delta \operatorname{Depth}_{i, j}\right|\right)
$$

where $\mathrm{j}$ denotes the locations of objects on the screen, and $\mathrm{N}$ is the total number of objects (2 or 4). Once beta values were obtained, we averaged betas across the two (or four) possible choice locations and also averaged betas across the two (or 12) not chosen locations (Fig. 1D, Supplementary Fig. 3D,E).

We also quantified the proportion of fits that produced significant values of each beta coefficient (Fig. 1E). The number of beta values significantly different from zero (alpha $=0.05)$ were summed across locations (2 or 4$)$ and across sessions. The results were then divided by the total number of beta values $(2$ * number of valid sessions or 4 * number of valid sessions, respectively). For not-chosen objects in the 4-object task, the number of significant fits were summed across three locations and then divided by 12 * number of valid sessions.

Depth-sign tuning and discrimination index. Average firing rates during stimulus presentation were plotted as a function of simulated depth (Fig. 2A-C) to construct depth tuning curves. To quantify the relative strength of neural responses to near and far depths defined by binocular disparity or motion parallax, we computed a depth-sign discrimination index (DSDI) from each tuning curve (Nadler et al. 2008; Nadler et al. 
2009).

$$
D S D I=\frac{1}{4} \sum_{i=1}^{4} \frac{R_{f a r(i)}-R_{\text {near }(i)}}{\left|R_{f a r(i)}-R_{\text {near }(i)}\right|+\sigma_{\operatorname{avg}(i)}}
$$

For each pair of depths symmetrical around zero (for example, \pm 2 deg), the difference in mean response between far $\left(R_{f a r}\right)$ and near $\left(R_{n e a r}\right)$ depths was computed relative to

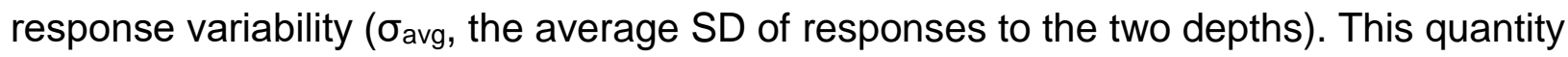
was then averaged across the four pairs of depth magnitudes to obtain the DSDI $(-1<$ DSDI <+1). Near-preferring neurons have negative DSDI values, whereas far-preferring neurons have positive DSDI values. Statistical significance of DSDI values was evaluated using a permutation test in which DSDI values were computed 1000 times after shuffling responses across depths. If the measured DSDI value is negative, the $p$ value is the proportion of shuffled DSDIs less than the measured DSDI value. If the measured DSDI is positive, the $p$ value is the proportion of DSDIs greater than the measured DSDI value.

Depth sign discrimination index for dynamic object tuning. Average firing rates during stimulus presentation were plotted as a function of depth difference (Fig. 2D-F) to construct dynamic object tuning curves. To quantify the relative strength of neural responses to negative and positive values of $\triangle$ Depth, we computed a DSDI metric for the dynamic object responses $\left(D S D I_{d y n}\right)$ as follows:

$$
D S D I_{d y n}=\frac{1}{3} \sum_{i=1}^{3} \frac{R_{\text {pos }(i)}-R_{n e g(i)}}{\left|R_{\text {pos }(i)}-R_{n e g}(i)\right|+\sigma_{\text {avg }(i)}}
$$

For each pair of $\Delta$ Depth values symmetrical around zero (e.g., $\pm 1.53 \mathrm{deg}$ ), the difference in mean response between positive $\left(R_{\text {pos }}\right)$ and negative $\left(R_{n e g}\right) \Delta$ Depth was computed relative to response variability ( $\sigma_{\mathrm{avg}}$, the average SD of responses to the two $\Delta$ Depth values). This quantity was then averaged across the three pairs of $\Delta$ Depth values to obtain $D S D I_{d y n}\left(-1<D S D I_{d y n}<+1\right)$.

Depth tuning congruency. Congruency of depth tuning curves obtained by manipulating binocular disparity and motion parallax cues was quantified using a correlation coefficient. The Pearson correlation was computed between the two cues using the average responses across nine depths (-2 to 2 deg in steps of $0.5 \mathrm{deg}$ ) for each cue; this coefficient is noted as RMP_BD (Fig. 3B). Neurons were classified as 
"congruent" or "opposite" if their value of RMP_BD was significantly greater or less than zero, respectively.

Neurometric performance. We used an ideal observer analysis to measure how reliably single neurons can signal whether an object is dynamic or stationary. For each value of $\Delta$ Depth, the distribution of firing rates across trials was sorted into two groups according to the type of object in the receptive field (dynamic vs. stationary). A receiver operating characteristic (ROC) curve was computed from the pair of response distributions for each $\Delta$ Depth (Britten et al. 1992), and performance of the ideal observer was defined as the area under the ROC curve. ROC areas were then plotted as a function of $\Delta$ Depth to construct a neurometric function (Fig. 5B, E). To obtain a single measure of neurometric performance (NP), we then averaged the ROC areas across nonzero values of $\Delta$ Depth to obtain a single metric for each neuron. This average $\mathrm{ROC}$ area will be $>0.5$ if a neuron responds preferentially to dynamic objects overall, and $<0.5$ if it responds preferentially to stationary objects overall.

Detection probability. Detection probability (DP) is a measure of the relationship between neural responses and perceptual decisions in a detection task (Bosking and Maunsell 2011), and is similar to the choice probability metric (Britten et al. 1996). The procedure for computing DP is analogous to the ROC analysis described above, except that responses are sorted into two groups according to the animal's perceptual decision (dynamic vs. stationary object in the receptive field). To eliminate any contamination from stimulus effects, only ambiguous trials ( $\Delta$ Depth $=0)$ were used to compute DP (Fig. 4). A permutation test was used to determine whether each DP value was significantly different from the chance level of 0.5 (Uka and DeAngelis 2004).

Decoding analyses. We constructed an optimal linear decoder to detect moving objects based on simulated responses from a population of 97 model neurons. Model neurons correspond to the dominant subset of recorded neurons for which data were collected under identical stimulus conditions, thus allowing us to construct pseudopopulation responses. We randomly selected 100,000 samples of stimulus conditions from the datasets with replacement (16 unique stimulus conditions within the RF). The mean and standard deviation of measured responses to each stimulus condition were 
then used to generate simulated responses according to the following equation (Cohen and Newsome 2009; Gu et al. 2014; Shadlen et al. 1996).

$$
\text { Response }=\mu+Q \times r_{\text {rand }} \times \sigma
$$

where $\mu$ and $\sigma$ are vectors of means and SDs of the population across stimulus conditions, $r_{\text {rand }}$ is a vector of standard normal deviates (MATLAB 'normrnd' function with zero mean and unity standard deviation), and $Q$ is the square root of the correlation matrix. The correlation matrix was modeled such that pairs of neurons with similar neurometric performance (NP) values have stronger correlated noise, and pairs of neurons with dissimilar NP values show weaker correlated noise:

$$
r_{-} \text {noise }_{i, j}=1.1 \times\left(0.5-\sqrt{\left|N P_{i}-N P_{j}\right|}\right)
$$

where $N P_{i}$ is the neurometric performance of neuron $\mathrm{i}$. This generated noise correlations $(0.15 \pm 0.17$, mean $\pm \mathrm{sd})$ of roughly similar strength to those observed in empirical studies of MT neurons (Huang and Lisberger 2009; Zohary et al. 1994).

Total trials were divided into training (90\%) and test (10\%) sets. A linear decoder was trained to classify whether the stimulus in the RF was a dynamic or stationary object based on population responses in the training set. We used linear discriminant analysis (MATLAB 'classify' function) to determine the weights of the decoder. Ambiguous trials $(\Delta$ Depth $=0)$ were excluded from the training set.

The test set was used to validate performance of the decoder. A predicted detection probability (DP pred) was computed for each neuron in the model in the same way we computed DP from the empirical data, except that the decoder's 'choice' for each trial was used instead of the monkey's behavioral choice. Specifically, responses to ambiguous stimuli $(\Delta$ Depth $=0)$ in the test set were sorted according to the decoder's output (dynamic vs. stationary object prediction).

Time course of choice-related responses. Spikes in the ambiguous trials $(\Delta \mathrm{Depth}$ $=0$ ) were aligned to stimulus onset, compiled into peri-stimulus time histograms, and then smoothed using a 150ms boxcar window. Trials were first sorted by the phase of self-motion (phase 0 or phase 180), and then sorted by the animal's choice (whether the animal chose an object within the receptive field or not). Average responses were zscored using a session-wide mean and standard deviation. We plotted the mean and 
standard error of the z-scored responses, as well as the difference in z-scored responses between choices (Supplementary Fig. 5). For each phase, we tested whether the median responses for the two choices at each time point were significantly different or not (alpha $=0.05$, Wilcoxon signed-rank test).

Neuron samples and selection criteria. We analyzed data from a total of 123 single units (53 from monkey 1, 70 from monkey 2) for which we completed the basic tuning measurements, including tuning for direction, speed, RF position, size, depth from binocular disparity, and depth from motion parallax. Among these, we completed the object detection task for 106 neurons (47 from monkey 1, 59 from monkey 2). This set of 106 neurons constitutes the sample for the single neuron analyses of Fig. 3. Except for two neurons, 104 of these 106 neurons were tested using a standard set of $\Delta$ Depth values, including zero (47 from monkey 1, 57 from monkey 2).

To compute detection probability, we analyzed a subset of these 104 neurons for which the monkey made at least five choices in favor of both target locations when $\Delta$ Depth=0 (92 neurons, 39 from monkey 1, 53 from monkey 2). For population decoding (Fig. 6), we required that each dataset contain responses to objects at all of the standard depth values for the stationary object. Three neurons were excluded because they were tested with slightly different stationary depth values, and 4 neurons were excluded because they did not have responses to stationary objects at all of the standard depth values (which can occur because the depths of stationary objects were chosen randomly from the standard values in each trial). Thus, with these exclusions, 97 neurons contributed to the population decoding analysis ( 45 from monkey 1, 52 from monkey 2).

\section{RESULTS}

We recorded from 123 well-isolated single neurons in area MT of two macaques that were trained to perform an object motion detection task during self-motion (53 neurons from monkey 1, 70 from monkey 2). We begin by describing the task and behavioral data, followed by analysis of the responses of isolated MT neurons during this task. Finally, we demonstrate that a simple linear decoder trained to perform the task based on responses of our MT population can recapitulate our main findings. 


\section{Stimulus configuration and behavioral task}

During neural recordings, monkeys viewed a display consisting of two square planar objects that were defined by random dot patterns (Fig. 1A, B; Supplementary Figure 1; Supplementary Video 1; see Methods for details). The animal viewed these objects while being translated $(0.5 \mathrm{~Hz}$ modified sinusoid, see Methods) along an axis in the fronto-parallel plane which corresponded with the preferred-null motion axis of the neuron under study. In the base condition of the task with no cue conflict between depth from disparity and motion parallax, both objects were simulated to be stationary in the world, such that their image motion was determined by the self-motion trajectory and the location of the objects in depth. When the objects were stationary in the world, their depth defined by motion parallax and disparity cues was the same, hence the difference in depth between the two cues was zero $\left(\Delta\right.$ Depth $\left.=d_{M P}-d_{B D}=0\right)$.

In other conditions ( $\Delta$ Depth $\neq 0)$, one of the objects was stationary in the world while the second "dynamic" object moved in space such that its depth defined by motion parallax, dMP, was not consistent with its depth defined by binocular disparity, dBD (Fig. 1B, Supplementary Fig. 1B; see Methods for details). As a result of this cue conflict between disparity and motion parallax, the dynamic object should appear to be moving in the world based on previous work in humans (Rushton et al. 2007). As $\Delta$ Depth becomes greater in magnitude, it should be easier for the animal to correctly determine which object is the dynamic object. Animals indicated their decision by making a saccade to one of two targets that appeared at the locations of the two objects at the end of the trial (Fig. 1A). Critically, due to the experimental design (see Methods for details), animals could not simply detect the dynamic object based on its retinal image velocity since the stationary object(s) in the display also moved on the retina due to selfmotion combined with depth variation.

Average psychometric functions for the two animals across 104 recording sessions are shown in Fig. 1C. As expected, the animals perform at chance when $\Delta$ Depth $=0$ and their percent correct increases with the magnitude of $\Delta$ Depth. This demonstrates that monkeys can perform the task as expected from human behavioral work (Rushton et al. 2007). Furthermore, we found that performance was very poor 
without binocular disparity cues (Supplementary Fig. 2), as also expected from previous work (Rushton et al. 2007).

The ranges of depths of the stationary and dynamic objects were overlapping but not identical (see Methods). To determine whether the animals primarily made their decisions based on $\Delta$ Depth, and not based upon the individual depths specified by disparity or motion parallax, we performed a logistic regression analysis to determine how animals perceptually weighted depth from motion parallax (|d $\mathrm{MP} \mid)$, depth from binocular disparity $\left(\left|\mathrm{d}_{\mathrm{BD}}\right|\right)$, and the magnitude of $\Delta$ Depth $\left(\left|d_{M P}-d_{B D}\right|\right.$; see Methods for details). Results show that animals primarily weighted the $\mid \Delta$ Depth| cue to make their decisions (Fig. 1D, E), although there were small contributions from the individual depth cues. We initially trained each animal to perform the task with 4 objects present in the display (3 stationary objects and 1 dynamic object), as well as three different pedestal depths, to make it more difficult for animals to rely on $\mathrm{d}_{\mathrm{MP}}$ or $\mathrm{d}_{\mathrm{BD}}$. Indeed, we found that the logistic regression weights were also strongly biased in favor of | $\Delta$ Depth| in the 4object version of the task (Supplementary. Fig. 3D,E). To increase the number of stimulus repetitions we could perform during recording experiments, we simplified the task to the two-object case.

Congruency of depth preferences and responses to dynamic objects

We measured the tuning of well-isolated MT neurons for depth defined by either binocular disparity or motion parallax cues, as described previously (Nadler et al. 2013, see also Methods). Receptive fields and direction preferences of the population of MT neurons are summarized in Supplementary Fig. 4. Fig. 2a shows data for a typical "congruent" cell, which prefers near depth defined by both disparity and motion parallax cues (see Methods for definition of congruent and opposite cells). Note that motion parallax stimuli are presented monocularly, such that selectivity for depth from motion parallax cannot be a consequence of binocular cues. In contrast, Fig. 2b,c show data for two example "opposite" cells that prefer near depths defined by motion parallax and moderate far depths defined by binocular disparity. Such neurons would, in principle, respond more strongly to some stimuli with discrepant disparity and motion parallax cues. Note that, for all of the example cells in Fig. 2a-c, responses to binocular disparity 
are substantially greater than responses to motion parallax. This is mainly because binocular disparity tuning was measured with constant-velocity stimuli at the preferred speed, whereas the range of speeds used to measure depth tuning based on motion parallax is generally lower (and covaries with depth magnitude).

As done previously (Kim et al. 2015a; 2017; 2015b; Nadler et al. 2008; Nadler et al. 2013; Nadler et al. 2009), we quantified the depth-sign preference of each MT neuron using a depth sign discrimination index (DSDI, see Methods), which takes on negative values for neurons with near preferences and positive values for neurons with far preferences. Across the population of 123 neurons, depth-sign preferences for motion parallax tended to be strongly biased toward near-preferring neurons, as reported previously (Nadler et al. 2008; Nadler et al. 2013), whereas depth-sign preferences for binocular disparity were rather well balanced (Fig. 3a). Importantly, there are roughly equal numbers of neurons in the lower-left and upper-left quadrants of Fig. 3a, indicating that congruent and opposite cells were roughly equally prevalent in our sample of MT neurons (see also Nadler et al. 2013). Thus, there are many opposite cells in MT that might respond selectively to dynamic objects over static objects.

Fig. $2 \mathrm{~d}$ shows responses of the example congruent cell (from Fig. 2a) that were obtained during the object detection task. Responses to the stationary object (red) are plotted as a function of the depth values specified by motion parallax (which are necessarily equal to binocular disparity values for a stationary object). Responses to the dynamic object (blue) are plotted as a function of both depth defined by motion parallax (lower abscissa) and depth defined by disparity (upper blue abscissa). This allows the reader to determine the depth value for each cue that is associated with a dynamic object having a particular $\Delta$ Depth value. For this example congruent cell (Fig. $2 d$ ), responses to stationary objects with large near depths substantially exceeded responses to any dynamic object.

A strikingly different pattern of results is seen for the example opposite cell in Fig. 2e. In this case, there are a few dynamic objects for which the neuron's response (blue) clearly exceeds the response to stationary objects of all different depth values (red). More specifically, this incongruent cell responds most strongly to dynamic objects that have large near depths defined by motion parallax and depths near the plane of fixation 
(0 deg) as defined by binocular disparity. This pattern of results is expected from the individual tuning curves in Fig. 2b, and demonstrates that this opposite cell is preferentially activated by a subset of dynamic objects. The second example opposite cell in Fig. 2c, f shows a generally similar pattern of results. For this cell, peak responses to stationary and dynamic objects are similar, but the neuron responds more strongly to dynamic objects over most of the stimulus range. Since we applied our $\Delta$ Depth manipulation around a fixed pedestal depth of -0.45 deg (to facilitate decoding, see Methods), we don't expect dynamic objects to preferentially activate every opposite cell. However, cells that are preferentially activated by dynamic objects should tend to be neurons with mismatched depth tuning for motion parallax and binocular disparity cues.

Fig. 3b shows that this expected relationship holds across our population of MT neurons. The ratio of peak responses for dynamic:stationary objects is plotted as a function of the correlation coefficient, RMP_BD, between depth tuning curves for disparity and motion parallax. Neurons with $\mathrm{R}_{\mathrm{MP}} \mathrm{BD}<0$ (opposite cells) tend to have peak response ratios that lie in the upper-left quadrant, indicating that opposite cells tend to be preferentially activated by dynamic objects. In contrast, neurons with $\mathrm{R}_{\mathrm{MP}} \mathrm{BD}>0$ (congruent cells), tend to have peak response ratios in the lower-right quadrant, indicating that they tend to be preferentially activated by stationary objects. Across the population, peak response ratio is significantly anti-correlated with $\operatorname{R}_{M P_{-} B D}(n=106$, Spearman rank correlation, $\mathrm{R}=-0.39, \mathrm{P}=2.8 \times 10^{-5}$ ), indicating that the hypothesized relationship between tuning congruency and response to scene-relative object motion is observed.

We further tested whether differences in depth tuning curves for binocular disparity and motion parallax can predict whether neurons prefer positive or negative $\Delta$ Depth values. Using responses to the dynamic object, we quantified each neuron's preference for positive/negative $\triangle$ Depth values using a variant of the DSDI metric,

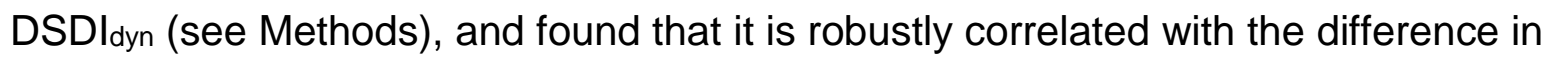
DSDI values ( $\triangle \mathrm{DSDI})$ computed from depth tuning curves for disparity and motion parallax (Fig. 3c, $\mathrm{R}=0.54, \mathrm{P}=2.7 \times 10^{-9}, \mathrm{n}=106$, Spearman correlation). Thus, selectivity for $\Delta$ Depth during the detection task is reasonably predictable from the congruency of depth tuning measured during a fixation task. 
Correlation with perceptual decisions

If neurons with mismatched depth tuning for disparity and motion parallax cues are selectively involved in detecting scene-relative object motion, we hypothesized that responses of these opposite cells would be correlated with the animals' perceptual decisions, whereas responses of congruent cells would not. To measure the correlation of neural activity with perceptual decisions, we took advantage of the fact that our design included a subset of trials in which both objects were stationary in the world and were presented at the pedestal depth of -0.45 deg (Fig. 4a). These conditions allowed us to quantify choice-related activity, for a fixed stimulus, by sorting responses into two groups: trials in which the monkey chose the object in the neuron's receptive field, and trials in which the monkey chose the object in the opposite hemi-field.

Data for an example neuron (Fig. 4b) show somewhat greater responses when the monkey chose the object located in the neuron's receptive field. We quantified this effect by applying ROC analysis to the two choice distributions (see Methods for details), which yielded a Detection Probability (DP) metric. DP will be greater than 0.5 when responses are greater on trials in which the monkey reported that the stimulus in the receptive field was the dynamic object. For the example neuron of Fig. $4 b$, the DP value was 0.75 , which is significantly greater than chance by permutation test $(p=$ 0.006, see Methods). Across a population of 92 neurons for which there were sufficient numbers of choices toward each stimulus (see Methods), the mean DP value of 0.56 was significantly greater than chance $\left(P=6.0 \times 10^{-5}, \mathrm{t}(91)=4.21, \mathrm{n}=92, t\right.$-test $)$ with 13/92 neurons showing individually significant DP values (Fig. 4c, filled bars). All neurons with significant DP values had effects in the expected direction, with DP $>0.5$. In addition, the mean DP value was significantly greater than chance for each monkey individually (monkey $1: \mathrm{n}=39$, mean $=0.59, \mathrm{P}=5.7 \times 10^{-4}, \mathrm{t}(38)=3.76$; monkey $2: \mathrm{n}=$ 53, mean $=0.53, P=0.03, t(52)=2.21$, $t$-test $)$.

Fig. 4 shows that many MT neurons have responses that are correlated with detection choices in the task. We hypothesized that neurons with DP $>0.5$ are more likely to be those that respond preferentially to dynamic objects over stationary objects. To obtain a signal-to-noise measure of each neuron's selectivity for dynamic vs. 
stationary objects, we again applied ROC analysis as illustrated for an opposite cell in Fig. 5a-c. This neuron responded more strongly to dynamic objects than stationary objects across most of the depth range (Fig. 5a). To quantify this selectivity, for each value of $\Delta$ Depth, responses were sorted into two groups: trials in which the dynamic object was in the receptive field, and trials in which the dynamic object was located in the opposite hemifield and a stationary object was in the receptive field (regardless of the depth of the stationary object). Thus, the ROC value computed for each $\Delta$ Depth value gave an indication of how well the neuron discriminated between that particular dynamic object and stationary objects of any depth. By convention, ROC values $>0.5$ indicate greater responses for a dynamic object in the receptive field.

Results of this analysis for the example opposite cell (Fig. 5b) show that ROC values were greater than 0.5 for all $\Delta$ Depth $\neq 0$; thus, this neuron reliably responded more strongly to dynamic objects than to stationary objects. To obtain a single metric for each neuron, we simply averaged the ROC metrics for each non-zero $\triangle$ Depth value, yielding a Neurometric Performance (NP) value of 0.78 for this neuron. The corresponding DP value for this neuron was 0.77 (Fig. 5c, $\mathrm{P}=0.0015$, permutation test), indicating that this neuron shows both strong selectivity for dynamic objects when $\Delta$ Depth $\neq 0$ and stronger responses when the animal reports a dynamic object in the receptive field when $\Delta$ Depth $=0$.

Data for an example congruent cell (Fig. 5d-f) show a very different pattern of results. This neuron generally responds more strongly to stationary objects of any depth than to dynamic objects (Fig. 5d). As a result, ROC values are consistently $<0.5$ when comparing responses to dynamic vs. stationary objects in the receptive field $(\Delta$ Depth $\neq 0$, Fig. $5 e$ ), yielding an NP value of 0.23 . The corresponding DP value for this neuron (Fig. 5f) was $0.39(P=0.26$, permutation test), indicating that it responded slightly more to ambiguous stimuli when the monkey reports that the object in the receptive field was stationary. Thus, the data from these two example neurons support the hypothesis that neurons with preferences for dynamic objects are selectively correlated with perceptual decisions.

To examine whether this hypothesis holds at the population level, we plotted the DP value for each neuron against the corresponding NP value. These two metrics, 
which are computed from completely different sets of trials ( $\Delta$ Depth $=0$ for $D P ; \Delta$ Depth $\neq 0$ for NP), are strongly correlated (Fig. $5 \mathrm{G}, \mathrm{R}=0.47, p=3.2 \times 10^{-6}, \mathrm{n}=92$, Spearman rank correlation) such that neurons with DP values substantially greater than 0.5 tend to be neurons that are selective for dynamic objects (NP > 0.5). In addition, we observed a significant positive correlation for each animal individually (monkey $1: n=39, R=0.59$, $\mathrm{P}=7.9 \times 10^{-5}$; monkey $2: \mathrm{n}=53, \mathrm{R}=0.33, \mathrm{P}=0.015$, Spearman correlation). It is also worth noting that all neurons with large DP values $(>0.7)$ also have NP values substantially greater than 0.5. Thus, the MT neurons that most strongly predict decisions to detect the dynamic object (on ambiguous trials) are those with incongruent tuning that makes them selective for dynamic objects.

We examined the time course of choice-related activity and found that it appeared within a few hundred milliseconds after the onset of self-motion (Supplementary Fig. 5). This choice-related activity was largely sustained throughout the rest of the stimulus period, even when motion of the object was in the anti-preferred direction.

\section{Decoding model}

The results described above suggest that perceptual detection of dynamic objects might be driven by the activity of neurons with incongruent tuning, which respond more strongly to dynamic objects. To further probe this hypothesis, we trained a simple linear decoder to detect dynamic objects based on simulated responses of a population of neurons that is closely based on our data, and we examined whether performance of the decoder shows a similar relationship between DP and NP (see Methods for details).

In the simulation (as in the experiments), the dynamic object could appear in either the right or left hemi-field, and the decoder was trained to report the location of the dynamic object. For each neuron in the population, responses were simulated to have the same mean and standard deviation as empirically measured responses. Since neurons were recorded separately and we could not measure correlated noise, we simulated responses based on either independent noise or correlated noise (see Methods for details). 
The decoder was trained to report the location of the dynamic object based on simulated population responses from the subset of trials for which $\Delta$ Depth $\neq 0$. The trained decoder was then used to predict responses for the completely ambiguous $(\Delta$ Depth $=0)$ trials in which identical objects were presented in both hemi-fields. Responses to ambiguous trials were then sorted according to the decoder output to compute predicted DP values (DP pred) for each neuron in the population.

We first compared DP pred with NP values for simulations in which all neurons were assumed to have independent noise. This decoder performs very well based on a sample of $97 \mathrm{MT}$ neurons (Fig. 6A, see Methods for selection criteria), indicating that there is extensive information available in a moderately-sized sample of MT neurons. We find a significant positive correlation between $\mathrm{DP}_{\text {pred }}$ and NP (Fig. 6B, $\mathrm{R}=0.53, \mathrm{P}=$ $4.9 \times 10^{-8}, \mathrm{n}=97$, Spearman correlation) in this simulation, consistent with the empirical observations of Fig. 5G. We also find a strong relationship (Fig. 6C, $R=0.92, P<1.0$ $\times 10^{-15}, \mathrm{n}=97$, Spearman correlation) between $\mathrm{DP}_{\text {pred }}$ and decoding weights, with positive readout weights being associated with $\mathrm{DP}_{\text {pred }}$ values greater than 0.5 .

While the relationship between DPpred and NP in Fig. 6B has a positive slope, DP pred values tend to be substantially closer to 0.5 than the values observed experimentally (Fig. 5G). However, this is not surprising given that neurons in this simulation were assumed to have independent noise. It is well established that neurons in MT exhibit correlated noise (e.g., Huang and Lisberger 2009; Zohary et al. 1994) and that choice-related activity is expected to be stronger in the presence of correlated noise (Britten et al. 1996; Gu et al. 2014; Haefner et al. 2013; Pitkow et al. 2015; Shadlen et al. 1996). Thus, we also simulated responses with a moderate level of correlated noise (median $R_{\text {noise }}=0.15$, see Methods for details), which had little impact on decoder performance (Fig. 6D). In the presence of correlated noise, DP pred values show a greater spread around 0.5, and are much more strongly correlated with NP values (Fig. $6 E, R=0.89, P<3.0 \times 10^{-16}, n=92$, Spearman correlation). While correlated noise enhances the relationship between $\mathrm{DP}_{\text {pred }}$ and NP, it also weakens the relationship between $\mathrm{DP}_{\text {pred }}$ and decoding weights (Fig. 6F, $\mathrm{R}=0.46, \mathrm{P}=3.4 \times 10^{-6}, \mathrm{n}=92$, Spearman correlation), as expected from theoretical studies (Haefner et al. 2013). 
Together, these simulations show that our main experimental finding is recapitulated by a simple linear decoder that is trained to distinguish between dynamic and static objects based on MT responses. Note, however, that we have not attempted to find parameters of our decoding simulations that would best match the empirical data (Fig. 5G). This would almost certainly be possible, but we don't feel that it is a worthwhile exercise given that we would have to make assumptions about the structure of correlated noise that we cannot sufficiently constrain. Nevertheless, these simulations demonstrate that a population of MT neurons with the depth tuning properties that we have described could be utilized to detect scene-relative object motion, and that such a read-out would produce the relationship between DP and NP that we have observed empirically.

\section{DISCUSSION}

We find that neurons having incongruent depth tuning for binocular disparity and motion parallax cues often respond more strongly to objects that move in the world than to stationary objects. Moreover, responses of cells with incongruent depth tuning more strongly predict perceptual decisions regarding object motion relative to the scene. While it has been established that humans can detect object motion based on cue conflicts between binocular disparity and motion parallax (Rushton et al. 2007), in the absence of other cues to object motion, the neural basis of this capacity has remained unknown. Our findings establish a simple neural mechanism for detecting moving objects, which has substantial advantages over other possible mechanisms (as discussed below) and likely complements them. In addition, our findings establish another important function for neurons with mismatched tuning for multiple stimulus cues, building on recent studies (Goncalves and Welchman 2017; Kim et al. 2016b; Sasaki et al. 2017; 2019; Zhang et al. 2019b). Our task involves a form of causal inference (Kording et al. 2007; Shams and Beierholm 2010), and our findings support the idea that a sensory representation consisting of a mixture of congruent and opposite cells provides a useful sensory substrate for causal inference (Rideaux et al. 2021). To our knowledge, these findings provide the first empirical evidence for a specific 
contribution of opposite neurons to perceptual inference about causes of sensory signals.

\section{Comparison to other types of mechanisms for detecting object motion}

In many instances, scene-relative object motion produces components of image motion that differ clearly in velocity or timing from the background optic flow at the corresponding location. Human observers can detect object motion when there are sufficient differences in local direction of motion between object and background (Royden and Connors 2010). Humans can also detect object motion based on local differences in speed when there are sufficient depth cues (Royden et al. 2016; Rushton et al. 2007) or when the image speed of an object is outside the range of background speeds in a particular task context (Royden and Moore 2012).

Royden and Holloway (2014) have shown that a model built on MT-like operators with surround suppression can effectively detect object motion when there are sufficient directional differences between object and background motion, or when object speed is outside the range of background speeds. However, such a model would not be able to detect object motion under task conditions like ours, or those of Rushton et al. (2007), because our dynamic object had the same motion axis as the stationary distractors and because the speeds of our dynamic objects were well within the range of speeds of stationary objects. More recently, Royden et al. (2015) have added disparity-tuned operators to their model, which allow detection of object motion even when it is aligned with background flow lines. This model computes local differences in response of separate velocity and disparity-tuned operators. It then applies an arbitrary threshold to detect cases for which there are differences and identifies these as possible object motion. While this model shows that differences in signals related to motion and disparity can be used to identify object motion in more general cases, it does not provide a biologically-plausible neural mechanism.

Our findings demonstrate a key, and apparently thus far unappreciated, neural mechanism for identifying local discrepancies between binocular disparity and motion parallax cues that accompany moving objects, even in difficult cases for which there are no local differences in the direction or timing of image motion. The activity of MT 
neurons with incongruent depth tuning for motion parallax and disparity provides a critical signal about these local discrepancies. Moreover, our simulations indicate that these signals can be easily read out by a linear decoder to detect object motion during self-motion.

Another approach to identifying scene-relative object motion during self-motion is flow parsing, in which global patterns of background motion related to self-motion are discounted (i.e., subtracted off) such that the remaining signal represents object motion relative to the scene (Rushton and Warren 2005). Several studies (Foulkes et al. 2013; Rushton et al. 2018; Warren and Rushton 2008; 2007; 2009b; Warren et al. 2012) have provided strong behavioral support for the flow-parsing hypothesis in humans, including some which suggest strongly that it involves a global motion process (Warren and Rushton 2009a). In addition, a recent study has demonstrated flow parsing in macaque monkeys (Peltier et al. 2020). If flow-parsing completely discounts background motion due to self-motion, then the computations for detecting scene-relative object motion would be greatly simplified and would be essentially the same as when there is no selfmotion. However, flow parsing alone may not be sufficient to detect scene-relative object motion. Recent evidence (Niehorster and Li 2017; Peltier et al. 2020) indicates that the gain of flow parsing can be well below unity, such that background motion is only partially discounted. In this case, the output of a flow-parsing mechanism would still not be sufficient to detect object motion, and a mechanism such as we found in area MT would still be very valuable.

An advantage of our proposed mechanism over flow parsing is that it does not require estimation of the global flow field, nor a complicated mechanism (Layton and Fajen 2020; 2016a) for implementing the flow parsing computation at each location in the visual field. Thus, our proposed mechanism may provide a valuable complement to flow parsing. Our results do not discount the contributions of center-surround or flowparsing mechanisms to detection of object motion, nor the contributions of mechanisms that may rely on multi-sensory signals (Kim et al. 2016b). Rather, our findings provide evidence of an additional complementary mechanism. Moreover, our findings establish the first direct (albeit correlational) evidence for a neural mechanism that is involved in perceptual dissociation of object and self-motion. 
Detecting scene-relative object motion is just one part of the computations needed to dissociate self-motion and object motion. For example, is also necessary to flexibly compensate for self-motion to compute object motion in different coordinate frames, such as head-centered or world-centered reference frames (Fajen et al. 2013; Sasaki et al. 2020). Some of these computations are likely to also rely on non-visual signals including vestibular signals about self-motion (Dokka et al. 2015a; Dokka et al. 2015b; Dokka et al. 2019; Fajen and Matthis 2013; Fajen et al. 2013; Sasaki et al. 2017; Sasaki et al. 2020). Thus, the mechanism proposed here is one part of a larger set of neural computations that remain to be fully understood.

\section{Functional roles of area MT and computational roles of opposite cells}

Area MT has traditionally been considered to hold a retinotopic representation of retinal image motion. Many studies still make this assumption, despite the fact that MT is known to be modulated by attention (Lee and Maunsell 2010; Martinez-Trujillo and Treue 2002; Treue and Maunsell 1996; 1999; Womelsdorf et al. 2008), eye movements (Bremmer et al. 1997; Chukoskie and Movshon 2009; Inaba et al. 2011; Inaba et al. 2007; Kim et al. 2017; Nadler et al. 2009; Newsome et al. 1988), and stimulus expectation (Schlack and Albright 2007). Our previous work has shown that MT neurons integrate retinal image motion with smooth eye movement (Nadler et al. 2008; Nadler et al. 2009) and global background motion (Kim et al. 2015b) signals to compute depth from motion parallax. In addition, most MT neurons are well known to be tuned for binocular disparity (DeAngelis and Newsome 1999; DeAngelis and Uka 2003; Maunsell and Van Essen 1983). Recent studies (Kim et al. 2015b; Nadler et al. 2013) revealed the existence of many MT neurons that have mismatched depth tuning for motion parallax and binocular disparity cues. Such neurons would presumably not be useful for cue integration in depth perception, and their functional role has thus far remained unclear. In the present study, we demonstrate that such "opposite" neurons provide valuable signals for detecting object motion during self-motion by selectively responding to local inconsistencies between binocular disparity and motion parallax cues. Thus, our findings provide novel evidence that the functional roles of MT go well beyond 
representing retinal image motion; they suggest that some MT neurons play fundamental roles in helping to infer the origins, or causes, of retinal image motion.

Our findings have parallels to the potential function of neurons in areas MSTd and VIP with mismatched heading tuning for visual and vestibular cues (Chen et al. 2013; 2011; Gu et al. 2008; Gu et al. 2006). Studies of cue integration and cue reweighting in heading perception have demonstrated that activity of congruent cells can account for behavioral performance (Fetsch et al. 2012; Gu et al. 2008), but the functional role of opposite cells remained unclear from those studies. More recent work has suggested that opposite neurons may play a role in helping parse the retinal image into signals related to self-motion and object motion (Kim et al. 2016b; Sasaki et al. 2017), although they did not link opposite cell activity to a relevant behavior. Thus, mismatched tuning, whether unisensory or multi-sensory, may be a common motif for performing computations that involve parsing sensory signals into components that reflect different causes in the world (Zhang et al. 2019b).

More generally, the parsing of retinal image motion into components related to object motion and self-motion is a causal inference problem (French and DeAngelis 2020; Kording et al. 2007; Shams and Beierholm 2010), and recent psychophysical work in humans has demonstrated that perception of heading in the presence of object motion follows predictions of a Bayesian causal inference model (Dokka et al. 2019). While the neural mechanisms of causal inference are still largely unknown (but see Fang et al. 2019), recent computational work has suggested that the relative activity of congruent and opposite cells may provide a critical signal for carrying out causal inference operations (Rideaux et al. 2021; Zhang et al. 2019a). By providing an empirical link between the activity of opposite cells and detection of object motion during self-motion, our results provide novel evidence for a sensory substrate that may be used to perform causal inference in the domain of object motion and self-motion perception. Elucidating the neural substrates and mechanisms of causal inference regarding object motion is the topic of ongoing studies in our laboratories. 


\section{FIGURE LEGENDS}

Figure 1. Object detection task and behavior. (A) Schematic Illustration of the moving object detection task. Once the animal fixated on a center target, objects were presented while the animal experienced self-motion. Saccade targets then appeared at the center of each object, and the animal indicated the dynamic object (moving relative to the scene) by making a saccade. (B) Schematic illustration of stimulus generation from behind and above the observer. A stationary far object that lies within the neuron's receptive field (RF, dashed circle) has rightward image motion when the observer moves to the right. The other (dynamic) object moves rightward independently in space (cyan arrow) such that the object's net motion suggests a far depth while binocular disparity cues suggest a near depth. Gray shaded region indicates the display screen; cross indicates the fixation point. (C) Average behavioral performance across recording sessions for each animal ( $n=47$ sessions from M1, $n=57$ sessions from M2, excluding two sessions for which the standard set of $\Delta$ Depth values was not used). Error bars denote $95 \%$ confidence intervals, which are generally smaller than the data points. (D) Normalized regression coefficients for depth from disparity $\left(\beta_{\mathrm{BD}}\right)$, depth from motion parallax $\left(\beta_{\mathrm{MP}}\right)$, and $\Delta$ Depth $\left(\beta_{\Delta}\right)$ are shown separately for chosen locations and notchosen locations (see text for details). Gray and black bars denote data for monkey 1 ( $n$ $=44)$ and monkey $2(n=53)$, respectively. (E) Proportion of fits for which each regression coefficient was significantly different from zero (alpha $=0.05$ ). Format as in panel D.

Figure 2. Responses of representative MT neurons. (A) Depth tuning curves for an example "congruent" neuron preferring near depths based on both binocular disparity (magenta) and motion parallax (cyan) cues (DSDIBD $=-0.81 ;$ DSDIMP $=-0.70 ; \mathrm{P}<0.05$ for both, permutation test; correlation $\left.\mathrm{R}_{\mathrm{MP}-\mathrm{BD}}=0.76, \mathrm{P}=0.016\right)$. Dashed horizontal lines indicate baseline activity for each tuning curve. (B) Tuning curves for an example "opposite" neuron preferring small far depths based on binocular disparity but preferring near depths based on motion parallax (DSDI $\mathrm{BD}=0.41$; DSDIMP $=-0.67 ; \mathrm{P}<0.05$ for both, permutation test; $\left.R_{M P-B D}=-0.36, P=0.32\right)$. $(C)$ Another example opposite cell preferring far depths based on binocular disparity but near depths based on motion 
parallax $\left(\mathrm{DSDI}_{\mathrm{BD}}=0.46\right.$; $\mathrm{DSDI} \mathrm{MP}_{\mathrm{MP}}=-0.56 ; \mathrm{P}<0.05$ for both, permutation test; $\mathrm{RMP}-\mathrm{BD}=-$ $0.73, P=0.025$ ) (D) Responses of the neuron in panel $A$ to stationary objects (red) and dynamic objects (blue) during performance of the detection task. Stationary objects were presented at various depths (bottom abscissa). Dynamic objects generally have conflicts $(\Delta$ Depth $\neq 0$ ) between depth from motion parallax (bottom abscissa) and binocular disparity (top abscissa). The pedestal depth at which $\Delta$ Depth $=0$ is shown as an unfilled blue triangle. (E) Responses during the detection task for the opposite cell of panel B. Format as in panel D. (F) Responses during detection for the neuron of panel C. Error bars in all panels represent s.e.m.

\section{Figure 3. Relationship between selectivity for moving objects and congruency} between depth cues. (A) Population summary of congruency of depth tuning for disparity and motion parallax. The DSDI value for binocular disparity tuning (DSDI $\mathrm{BD}$ ) is plotted as a function of the DSDI value for motion parallax tuning (DSDIMP) for each neuron $(n=123)$. Squares and triangles denote data for monkey $1(n=53)$ and monkey $2(n=70)$, respectively. (B) Population summary of relationship between relative responses to dynamic and stationary objects as a function of depth tuning congruency. The ordinate shows the ratio of peak responses for dynamic:stationary stimuli. The abscissa shows the correlation coefficient (RMP-BD) between depth tuning for motion parallax and disparity. Dashed line is a linear fit using type 2 regression $(n=106 ; n=47$ from monkey 1 and $n=59$ from monkey 2; sample includes all neurons for which we completed the detection task). (C) Population summary $(n=106)$ of the relationship between the preference for $\Delta$ Depth of the dynamic object (as quantified by $D S D I_{d y n}$, see Methods) and the difference between DSDIMP and DSDIBD.

Figure 4. Relationship between MT responses and detection of object motion. (A) When $\Delta$ Depth $=0$, two stationary objects at the pedestal depth had identical retinal motion and depth cues. Animals still were required to report one of the objects as dynamic. (B) To compute detection probability (DP), responses to the $\Delta$ Depth $=0$ condition were z-scored and sorted into two groups according to the animal's choice. Filled and open bars show distributions of z-scored responses of an example MT 
neuron when the animal reported that the moving object was in and out of the receptive field, respectively. (C) Distribution of DP values for a sample of 92 MT neurons, including all neurons tested in the detection task for which the animal made at least 5 choices in each direction (see Methods). Arrowhead shows the mean DP value of 0.56 , which was significant greater than $0.5\left(P=6.0 \times 10^{-5}, \mathrm{n}=92, t\right.$-test $)$.

\section{Figure 5. Relationship between detection probability and neurometric}

performance for dynamic objects. (A) Responses of an example opposite neuron to dynamic and stationary objects during the detection task. Format as in Fig. 2D. (B) ROC values comparing responses to a dynamic object at each value of $\Delta$ Depth with responses to stationary objects, for the neuron of panel A. Neurometric performance (NP $=0.78$ for this neuron) is defined as the average ROC area for all $\triangle$ Depth $\neq 0$. (C) Distribution of z-scored responses sorted by choice for the same neuron as in panels A,B. Format as in Fig. 4B. (D-F) Data from an example congruent cell, plotted in the same format as panels A-C. (G) Relationship between DP and NP for a population of 92 MT neurons. Dashed line: linear fit using type 2 regression (slope $=1.06$, slope $\mathrm{Cl}=$ [0.80 1.48]; intercept $=-0.04$, intercept $\mathrm{Cl}=\left[\begin{array}{lll}-0.31 & 0.11\end{array}\right]$ ).

\section{Figure 6. Linear decoding reproduces the relationship between Detection}

Probability and Neurometric Performance. (A) Performance of a linear decoder that was trained to detect moving objects based on simulated population responses with independent noise (see Methods for details). Error bars represent $95 \%$ Cls ( $n=100$ simulations). (B) Neural responses were sorted by the output of the decoder to compute a predicted detection probability ( $\left.\mathrm{DP}_{\text {pred }}\right)$ for each unit in the simulated population $(\mathrm{N}=97$, including all neurons recorded in the detection task using identical $\Delta$ Depth and stationary depth values, see Methods). DP pred is plotted as a function of the measured Neurometric Performance (NP) for each neuron. Error bars represent $95 \%$ Cls ( $n=100$ simulations). (C) Relationship between $\mathrm{DP}_{\text {pred }}$ and the readout weight $(\beta)$ for each unit in the decoded population. Error bars represent 95\% Cls. (D-F) Analogous results for a decoder that was trained based on population responses with modest correlated noise (see text and Methods for details). Format as in panels A-C. 
Supplementary Figure 1. Visual display and motion trajectories. (A) A screen shot of the visual stimulus. It consisted of fixation target at the center, two or four objects (two objects shown), and background dots, which were masked within the central region of the display. Red and green dots represent images shown to the left and right eyes. (B) Schematic drawing (top view) depicting the rendering geometry for a far stationary object (left) and a near dynamic object (right). Left: the location of a stationary object was initially defined by the horizontal and vertical coordinates on the screen, and then was ray-traced onto a virtual plane located at the depth of the object. A moving object was initially positioned at the depth defined by binocular disparity ( $\mathrm{d}_{\mathrm{BD}}$, cyan) in the same way as the stationary object. The location of the object at a different depth (dMP) was ray-traced (blue star). Right: once the self-motion trajectory of the animal was determined, the location of the virtual object (blue star) was ray-traced onto the plane defined by binocular disparity $\left(\mathrm{d}_{\mathrm{BD}}\right)$ to compute the trajectory of independent movement at $\mathrm{d}_{\mathrm{BD}}$. The resultant retinal motion mimics motion parallax at depth $\mathrm{dmP}$. We used the depth difference ( $\Delta$ Depth) to manipulate the difficulty of the task. (C) Time courses of position (left) and velocity (right) of the animal during the modified sinusoidal translational motion (see Methods for details).

\section{Supplementary Figure 2. Results from a control experiment including} monocularly presented objects. Psychometric data are shown from control experiments ( $\mathrm{N}=3$ sessions, 1180 total trials) in which dynamic and stationary objects were shown with (binocular) and without (monocular) disparity cues. Monocular conditions were only presented for the largest (easiest) $\Delta$ Depth values $(16 \%$ of total trials). While performance in the binocular condition is comparable to the main dataset (Fig. 1C), performance in the monocular condition is very poor. Error bars represent $95 \%$ Cls.

\section{Supplementary Figure 3. Behavioral performance in the more generalized task} with 4 objects. (A) An example session from monkey 1 in which the animal performed the detection task with 4 objects and 3 pedestal depths (red, green, and blue colors). (B) 
An example session from monkey 2 prior to neural recordings. (C) Another example session from monkey 2 after neural recording experiments were completed. (D) Normalized beta coefficients from logistic regression analysis for the four-object task; format as in Fig. 1D. Data include 35 sessions from monkey 1 from before neural recordings, 27 sessions from monkey 2 from before neural recordings, and 10 sessions from monkey 2 after neural recordings. (E) Proportion of beta coefficients that are significantly different from zero. Format as in Fig. 1E.

Supplementary Figure 4. Distribution of receptive field properties. (A) Positions and sizes of the receptive fields of our sample of MT neurons $(n=123)$. Each circle represents the contour of the receptive field, defined as the center and diameter obtained from the receptive field mapping and size tuning protocols (see Methods). (B) Distribution of preferred directions for the sample of neurons ( $n=123)$, where 0 deg corresponds to rightward motion and 90 deg corresponds to upward motion.

\section{Supplementary Figure 5. Time courses of choice-related activity. Average}

population responses in the ambiguous trials $(\Delta$ Depth $=0)$ sorted by the animal's choice. Neurons showing positive DP values were analyzed ( $n=64$ neurons). (A) Average time courses of z-scored responses for the subset of trials in which self-motion began toward the neuron's preferred direction (phase 0). We first computed the moving averages of spiking activity (150 ms window) for each neuron. The results were then zscored based on the mean and standard deviation of the moving averages for each neuron. (B) Responses when self-motion began toward the neuron's anti-preferred direction (phase 180). (C) The differential response between the two choice groups shown in panel A. Gray marks denote time points at which the differential response is significantly different from zero (alpha $=0.05, \mathrm{n}=64$, Wilcoxon signed-rank test). (D) Differential response between the two choice groups shown in panel B. Shadings represent s.e.m. 


\section{Supplementary Video 1. Visual stimuli used in the dynamic object detection task.}

Examples of visual stimuli in the two-object task, assuming that the receptive field of a neuron is located on the horizontal meridian. The video shows a sequence of seven stimuli, which are sorted by their $\Delta$ Depth values $(\Delta$ Depth $=-1.53,-0.57,-0.21,0,0.21$, 0.57 , and $1.53 \mathrm{deg}$ ). The depth of the stationary object in each stimulus is labelled and was chosen randomly. In the actual experiment, the fixation target was stationary in the world, and the motion platform moved the animal and screen sinusoidally along an axis in the frontoparallel plane (here a horizontal axis). Thus, the video shows the scene from the viewpoint of the moving observer. The stimulus sequences are equivalent to a situation in which the observer remains stationary and the entire scene is translated in front of the observer. For each $\Delta$ Depth value, two full cycles of the stimulus are shown for display purposes; in the actual experiment, each trial consisted of just one cycle. During the second cycle of each stimulus in the video, the text label indicates whether the dynamic object was on the left or right side of the display. Red and green dots in the video denote the stereo half-images for the left and right eyes. Note that, without viewing the images stereoscopically and tracking the fixation target, it is generally not possible to determine the location of the dynamic object from the image motion on the display. 


\section{REFERENCES}

Albright TD, Desimone R, and Gross CG. Columnar organization of directionally selective cells in visual area MT of the macaque. Journal of neurophysiology 51: 16-31, 1984.

Bosking WH, and Maunsell $\mathbf{J H}$. Effects of stimulus direction on the correlation between behavior and single units in area MT during a motion detection task. The Journal of neuroscience : the official journal of the Society for Neuroscience 31: 82308238, 2011.

Bremmer F, Ilg UJ, Thiele A, Distler C, and Hoffmann KP. Eye position effects in monkey cortex. I. Visual and pursuit-related activity in extrastriate areas MT and MST. Journal of neurophysiology 77: 944-961, 1997.

Britten KH, Newsome WT, Shadlen MN, Celebrini S, and Movshon JA. A relationship between behavioral choice and the visual responses of neurons in macaque MT. Visual neuroscience 13: 87-100, 1996.

Britten KH, Shadlen MN, Newsome WT, and Movshon JA. The analysis of visual motion: a comparison of neuronal and psychophysical performance. The Journal of neuroscience : the official journal of the Society for Neuroscience 12: 4745-4765, 1992. Chen A, Deangelis GC, and Angelaki DE. Functional specializations of the ventral intraparietal area for multisensory heading discrimination. The Journal of neuroscience : the official journal of the Society for Neuroscience 33: 3567-3581, 2013.

Chen A, DeAngelis GC, and Angelaki DE. Representation of vestibular and visual cues to self-motion in ventral intraparietal cortex. The Journal of neuroscience : the official journal of the Society for Neuroscience 31: 12036-12052, 2011.

Chukoskie L, and Movshon JA. Modulation of visual signals in macaque MT and MST neurons during pursuit eye movement. Journal of neurophysiology 102: 3225-3233, 2009.

Cohen MR, and Newsome WT. Estimates of the contribution of single neurons to perception depend on timescale and noise correlation. The Journal of neuroscience : the official journal of the Society for Neuroscience 29: 6635-6648, 2009.

DeAngelis GC, and Newsome WT. Organization of disparity-selective neurons in macaque area MT. J Neurosci 19: 1398-1415, 1999.

DeAngelis GC, and Uka T. Coding of horizontal disparity and velocity by MT neurons in the alert macaque. Journal of neurophysiology 89: 1094-1111, 2003.

Dokka K, DeAngelis GC, and Angelaki DE. Multisensory Integration of Visual and Vestibular Signals Improves Heading Discrimination in the Presence of a Moving Object. The Journal of neuroscience : the official journal of the Society for Neuroscience 35: 13599-13607, 2015a.

Dokka K, MacNeilage PR, DeAngelis GC, and Angelaki DE. Multisensory self-motion compensation during object trajectory judgments. Cereb Cortex 25: 619-630, 2015b.

Dokka K, Park H, Jansen M, DeAngelis GC, and Angelaki DE. Causal inference accounts for heading perception in the presence of object motion. Proc Natl Acad Sci U S A 116: 9060-9065, 2019.

Fajen BR, and Matthis JS. Visual and non-visual contributions to the perception of object motion during self-motion. PLoS One 8: e55446, 2013.

Fajen BR, Parade MS, and Matthis JS. Humans perceive object motion in world coordinates during obstacle avoidance. Journal of vision 13: 2013. 
Fang W, Li J, Qi G, Li S, Sigman M, and Wang L. Statistical inference of body representation in the macaque brain. Proc Natl Acad Sci U S A 116: 20151-20157, 2019.

Fetsch CR, Pouget A, DeAngelis GC, and Angelaki DE. Neural correlates of reliability-based cue weighting during multisensory integration. Nature neuroscience 15: 146-154, 2012.

Foulkes AJ, Rushton SK, and Warren PA. Flow parsing and heading perception show similar dependence on quality and quantity of optic flow. Front Behav Neurosci 7: 49, 2013.

French RL, and DeAngelis GC. Multisensory neural processing: from cue integration to causal inference. Current Opinion in Physiology 16: 8-13, 2020.

Gibson EJ, Gibson JJ, Smith OW, and Flock H. Motion parallax as a determinant of perceived depth. Journal of experimental psychology 58: 40-51, 1959.

Goncalves NR, and Welchman AE. "What Not" Detectors Help the Brain See in Depth. Current biology : CB 27: 1403-1412 e1408, 2017.

Gu Y, Angelaki DE, and DeAngelis GC. Contribution of correlated noise and selective decoding to choice probability measurements in extrastriate visual cortex. eLife 3: 2014. Gu Y, Angelaki DE, and Deangelis GC. Neural correlates of multisensory cue integration in macaque MSTd. Nature neuroscience 11: 1201-1210, 2008.

Gu Y, Watkins PV, Angelaki DE, and DeAngelis GC. Visual and nonvisual contributions to three-dimensional heading selectivity in the medial superior temporal area. The Journal of neuroscience : the official journal of the Society for Neuroscience 26: 73-85, 2006.

Haefner RM, Gerwinn S, Macke JH, and Bethge M. Inferring decoding strategies from choice probabilities in the presence of correlated variability. Nature neuroscience 16: 235-242, 2013.

Huang X, and Lisberger SG. Noise correlations in cortical area MT and their potential impact on trial-by-trial variation in the direction and speed of smooth-pursuit eye movements. Journal of neurophysiology 101: 3012-3030, 2009.

Inaba N, Miura K, and Kawano K. Direction and speed tuning to visual motion in cortical areas MT and MSTd during smooth pursuit eye movements. Journal of neurophysiology 105: 1531-1545, 2011.

Inaba N, Shinomoto S, Yamane S, Takemura A, and Kawano K. MST neurons code for visual motion in space independent of pursuit eye movements. Journal of neurophysiology 97: 3473-3483, 2007.

Kim HR, Angelaki DE, and DeAngelis GC. A functional link between MT neurons and depth perception based on motion parallax. The Journal of neuroscience : the official journal of the Society for Neuroscience 35: 2766-2777, 2015a.

Kim HR, Angelaki DE, and DeAngelis GC. Gain Modulation as a Mechanism for Coding Depth from Motion Parallax in Macaque Area MT. The Journal of neuroscience : the official journal of the Society for Neuroscience 37: 8180-8197, 2017.

Kim HR, Angelaki DE, and DeAngelis GC. The neural basis of depth perception from motion parallax. Philos Trans $R$ Soc Lond B Biol Sci 371: 2016a.

Kim HR, Angelaki DE, and DeAngelis GC. A novel role for visual perspective cues in the neural computation of depth. Nature neuroscience 18: 129-137, 2015b. 
Kim HR, Pitkow X, Angelaki DE, and DeAngelis GC. A simple approach to ignoring irrelevant variables by population decoding based on multisensory neurons. Journal of neurophysiology 116: 1449-1467, 2016b.

Koenderink JJ, and van Doorn AJ. Facts on optic flow. Biological cybernetics 56: 247254, 1987.

Komatsu H, and Wurtz RH. Relation of cortical areas MT and MST to pursuit eye movements. I. Localization and visual properties of neurons. Journal of neurophysiology 60: 580-603, 1988.

Kording KP, Beierholm U, Ma WJ, Quartz S, Tenenbaum JB, and Shams L. Causal inference in multisensory perception. PLoS One 2: e943, 2007.

Layton OW, and Fajen BR. Computational Mechanisms for Perceptual Stability using Disparity and Motion Parallax. The Journal of neuroscience : the official journal of the Society for Neuroscience 40: 996-1014, 2020.

Layton OW, and Fajen BR. A Neural Model of MST and MT Explains Perceived Object Motion during Self-Motion. The Journal of neuroscience : the official journal of the Society for Neuroscience 36: 8093-8102, 2016a.

Layton OW, and Fajen BR. The temporal dynamics of heading perception in the presence of moving objects. Journal of neurophysiology 115: 286-300, 2016b.

Lee $\mathbf{J}$, and Maunsell JH. Attentional modulation of MT neurons with single or multiple stimuli in their receptive fields. The Journal of neuroscience : the official journal of the Society for Neuroscience 30: 3058-3066, 2010.

Martinez-Trujillo $\mathrm{J}$, and Treue S. Attentional modulation strength in cortical area MT depends on stimulus contrast. Neuron 35: 365-370, 2002.

Maunsell JH, and Van Essen DC. Functional properties of neurons in middle temporal visual area of the macaque monkey. II. Binocular interactions and sensitivity to binocular disparity. J Neurophysiol 49: 1148-1167, 1983.

Nadler JW, Angelaki DE, and DeAngelis GC. A neural representation of depth from motion parallax in macaque visual cortex. Nature 452: 642-645, 2008.

Nadler JW, Barbash D, Kim HR, Shimpi S, Angelaki DE, and DeAngelis GC. Joint representation of depth from motion parallax and binocular disparity cues in macaque area MT. The Journal of neuroscience : the official journal of the Society for Neuroscience 33: 14061-14074, 14074a, 2013.

Nadler JW, Nawrot M, Angelaki DE, and DeAngelis GC. MT neurons combine visual motion with a smooth eye movement signal to code depth-sign from motion parallax. Neuron 63: 523-532, 2009.

Newsome WT, Wurtz RH, and Komatsu H. Relation of cortical areas MT and MST to pursuit eye movements. II. Differentiation of retinal from extraretinal inputs. Journal of neurophysiology 60: 604-620, 1988.

Niehorster DC, and Li L. Accuracy and Tuning of Flow Parsing for Visual Perception of Object Motion During Self-Motion. Iperception 8: $2041669517708206,2017$.

Peltier NE, Angelaki DE, and DeAngelis GC. Optic flow parsing in the macaque monkey. Journal of vision In Press: 2020.

Pitkow X, Liu S, Angelaki DE, DeAngelis GC, and Pouget A. How Can Single Sensory Neurons Predict Behavior? Neuron 87: 411-423, 2015.

Rideaux R, Storrs KR, Maiello G, and Welchman AE. How multisensory neurons solve causal inference. Proc Natl Acad Sci U S A 118: 2021. 
Royden CS, and Connors EM. The detection of moving objects by moving observers. Vision research 50: 1014-1024, 2010.

Royden CS, and Holloway MA. Detecting moving objects in an optic flow field using direction- and speed-tuned operators. Vision research 98: 14-25, 2014.

Royden CS, and Moore KD. Use of speed cues in the detection of moving objects by moving observers. Vision research 59: 17-24, 2012.

Royden CS, Parsons D, and Travatello J. The effect of monocular depth cues on the detection of moving objects by moving observers. Vision research 124: 7-14, 2016.

Royden CS, Sannicandro SE, and Webber LM. Detection of moving objects using motion- and stereo-tuned operators. Journal of vision 15: 21, 2015.

Rushton SK, Bradshaw MF, and Warren PA. The pop out of scene-relative object movement against retinal motion due to self-movement. Cognition 105: 237-245, 2007. Rushton SK, Niehorster DC, Warren PA, and Li L. The Primary Role of Flow Processing in the Identification of Scene-Relative Object Movement. The Journal of neuroscience : the official journal of the Society for Neuroscience 38: 1737-1743, 2018. Rushton SK, and Warren PA. Moving observers, relative retinal motion and the detection of object movement. Current biology : CB 15: R542-543, 2005.

Sasaki R, Angelaki DE, and DeAngelis GC. Dissociation of Self-Motion and Object Motion by Linear Population Decoding That Approximates Marginalization. The Journal of neuroscience : the official journal of the Society for Neuroscience 37: 11204-11219, 2017.

Sasaki R, Angelaki DE, and DeAngelis GC. Processing of object motion and selfmotion in the lateral subdivision of the medial superior temporal area in macaques. Journal of neurophysiology 121: 1207-1221, 2019.

Sasaki R, Anzai A, Angelaki DE, and DeAngelis GC. Flexible coding of object motion in multiple reference frames by parietal cortex neurons. Nature neuroscience 23: 10041015, 2020.

Schlack A, and Albright TD. Remembering visual motion: neural correlates of associative plasticity and motion recall in cortical area MT. Neuron 53: 881-890, 2007. Shadlen MN, Britten KH, Newsome WT, and Movshon JA. A computational analysis of the relationship between neuronal and behavioral responses to visual motion. The Journal of neuroscience : the official journal of the Society for Neuroscience 16: 14861510, 1996.

Shams L, and Beierholm UR. Causal inference in perception. Trends Cogn Sci 14: 425-432, 2010.

Treue S, and Maunsell JH. Attentional modulation of visual motion processing in cortical areas MT and MST. Nature 382: 539-541, 1996.

Treue S, and Maunsell JH. Effects of attention on the processing of motion in macaque middle temporal and medial superior temporal visual cortical areas. The Journal of neuroscience : the official journal of the Society for Neuroscience 19: 7591-7602, 1999. Uka T, and DeAngelis GC. Contribution of area MT to stereoscopic depth perception: choice-related response modulations reflect task strategy. Neuron 42: 297-310, 2004. Van Essen DC, Drury HA, Dickson J, Harwell J, Hanlon D, and Anderson CH. An integrated software suite for surface-based analyses of cerebral cortex. J Am Med Inform Assoc 8: 443-459, 2001. 
Warren PA, and Rushton SK. Evidence for flow-parsing in radial flow displays. Vision research 48: 655-663, 2008.

Warren PA, and Rushton SK. Optic flow processing for the assessment of object movement during ego movement. Current biology : CB 19: 1555-1560, 2009a.

Warren PA, and Rushton SK. Perception of object trajectory: parsing retinal motion into self and object movement components. Journal of vision 7: 2 1-11, 2007.

Warren PA, and Rushton SK. Perception of scene-relative object movement: Optic flow parsing and the contribution of monocular depth cues. Vision research 49: $1406-$ 1419, 2009b.

Warren PA, Rushton SK, and Foulkes AJ. Does optic flow parsing depend on prior estimation of heading? Journal of vision 12: 8, 2012.

Womelsdorf T, Anton-Erxleben K, and Treue S. Receptive field shift and shrinkage in macaque middle temporal area through attentional gain modulation. The Journal of neuroscience : the official journal of the Society for Neuroscience 28: 8934-8944, 2008. Zhang W, Wu S, Doiron B, and Lee TS. A Normative Theory for Causal Inference and Bayes Factor Computation in Neural Circuits. bioRxiv 3799--3808, 2019a.

Zhang WH, Wang H, Chen A, Gu Y, Lee TS, Wong KM, and Wu S. Complementary congruent and opposite neurons achieve concurrent multisensory integration and segregation. eLife 8: 2019b.

Zohary E, Shadlen MN, and Newsome WT. Correlated neuronal discharge rate and its implications for psychophysical performance. Nature 370: 140-143, 1994. 

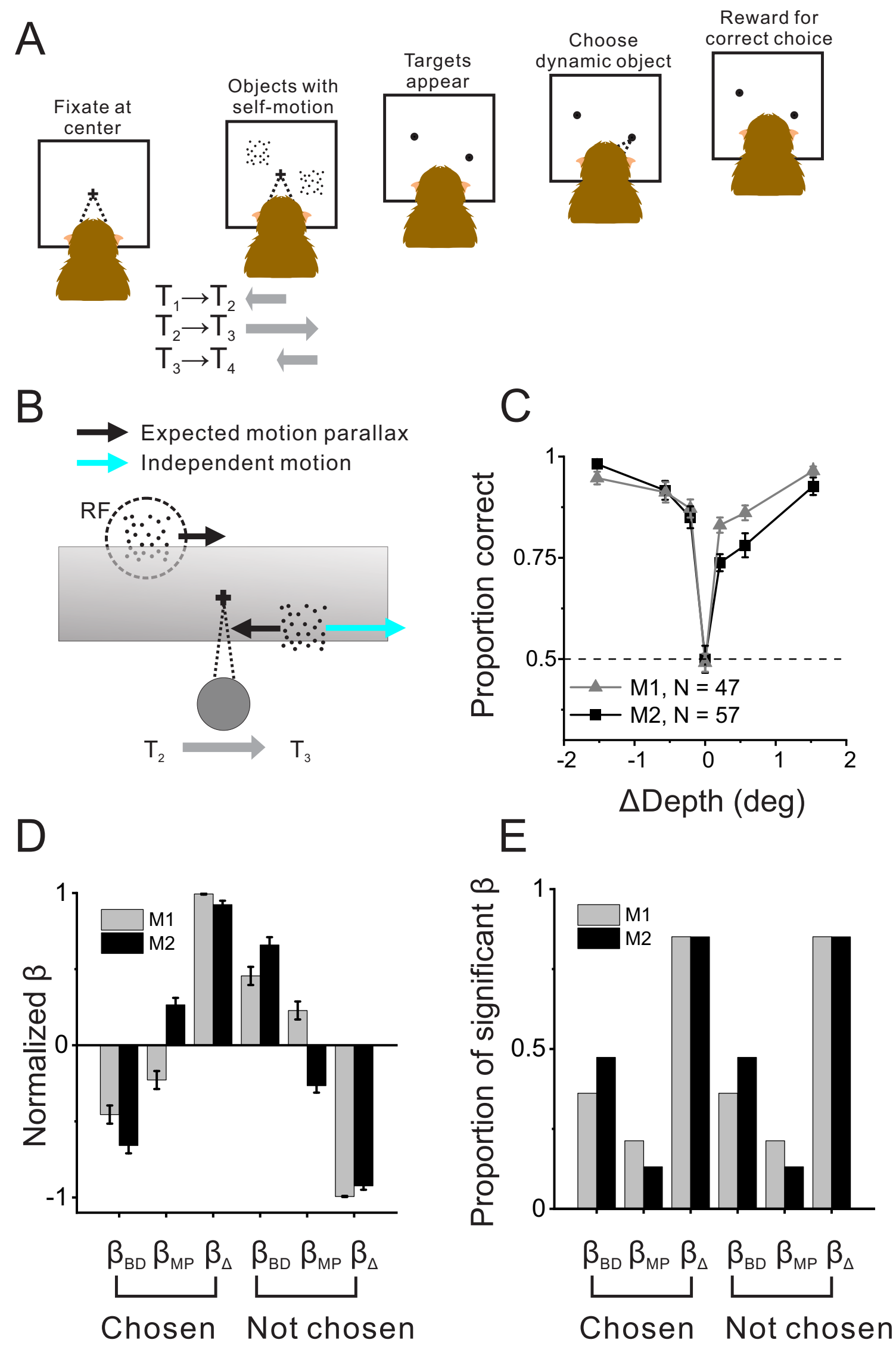

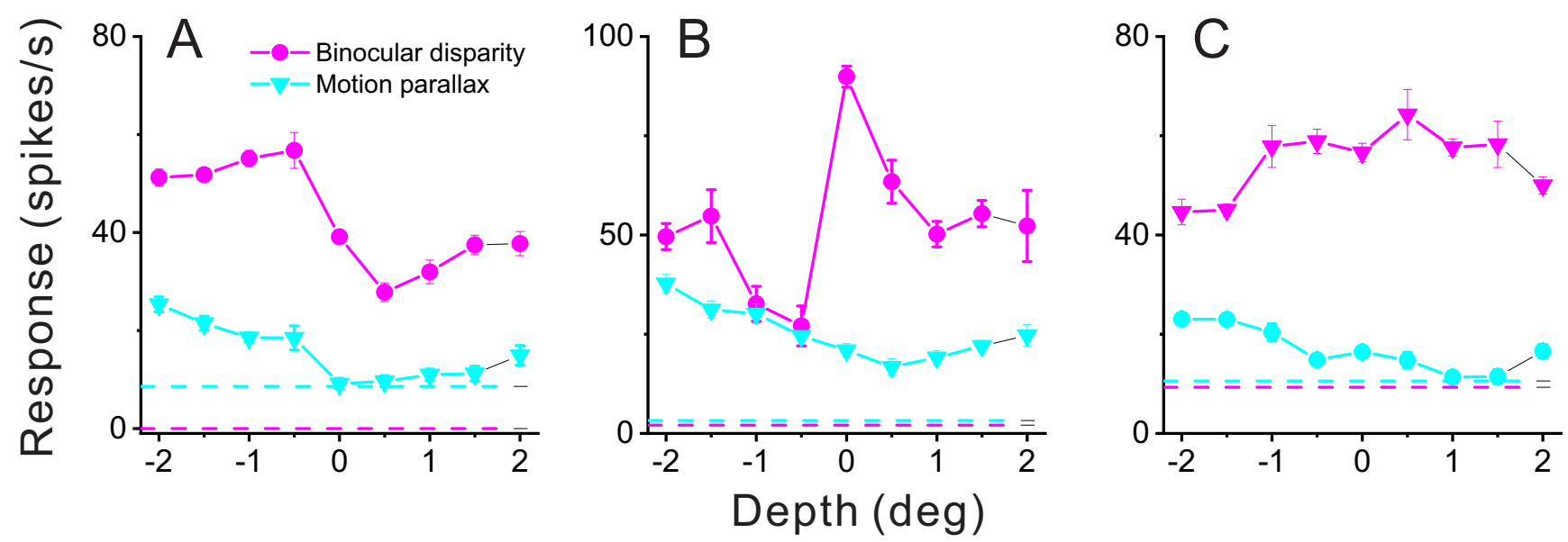

Depth from binocular disparity (deg)
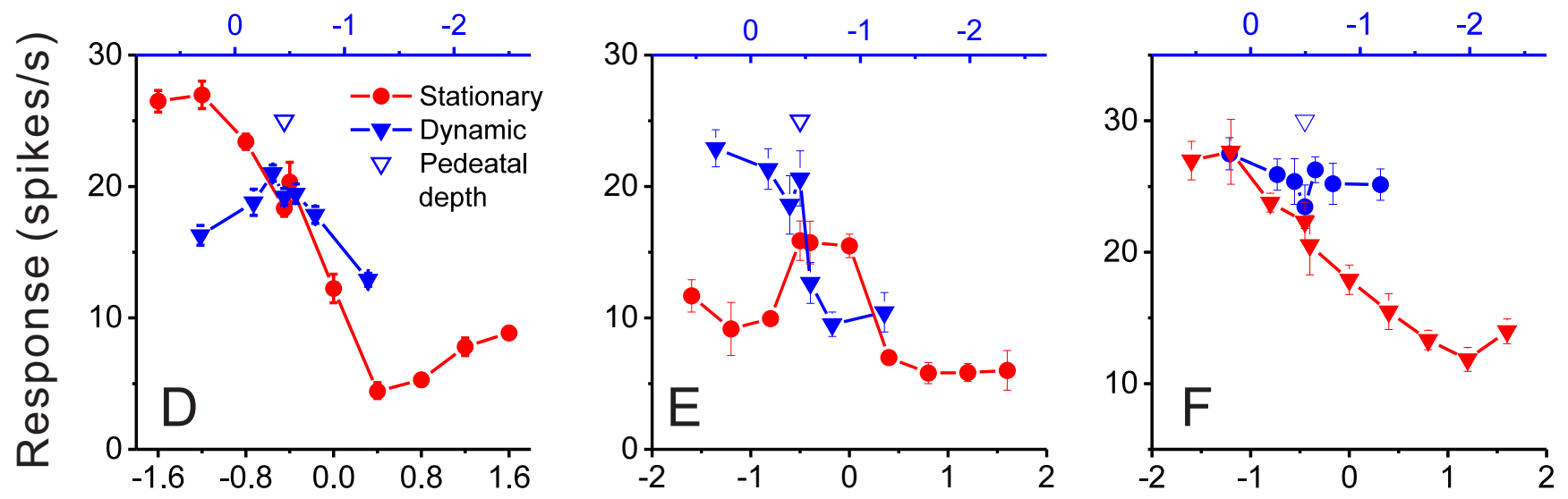

Depth from motion parallax (deg) 

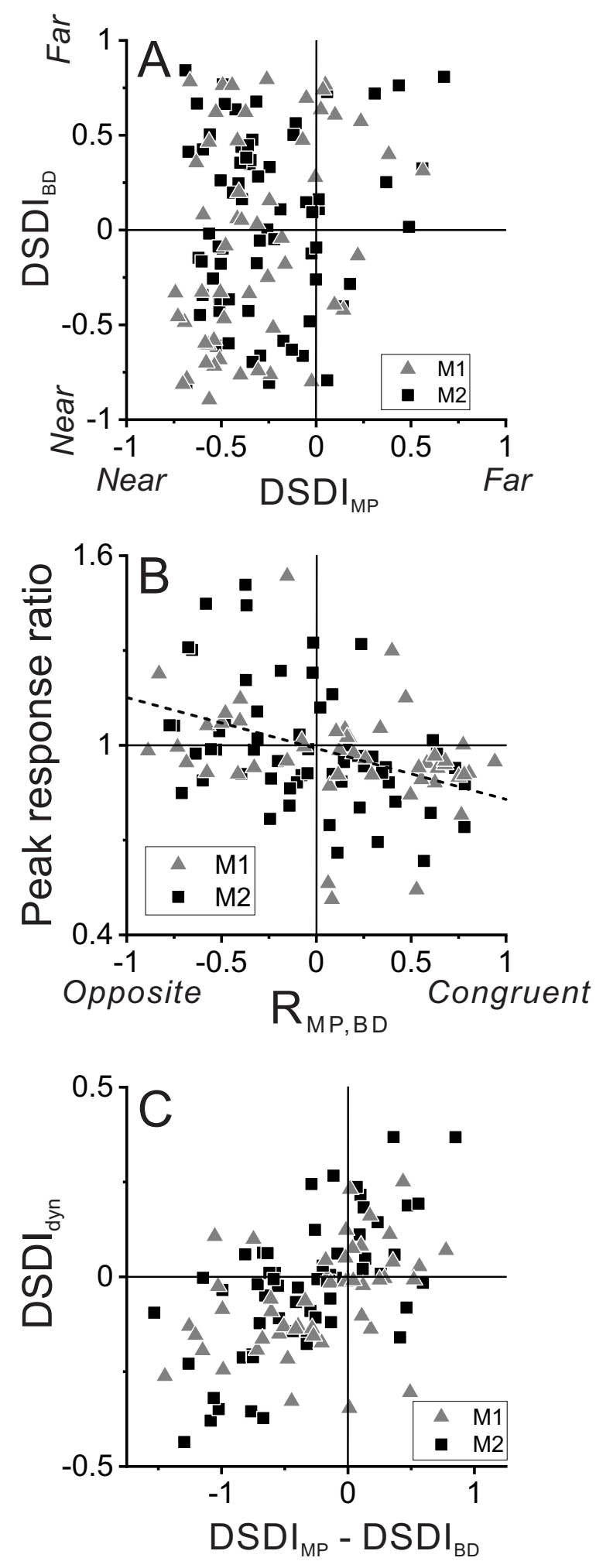

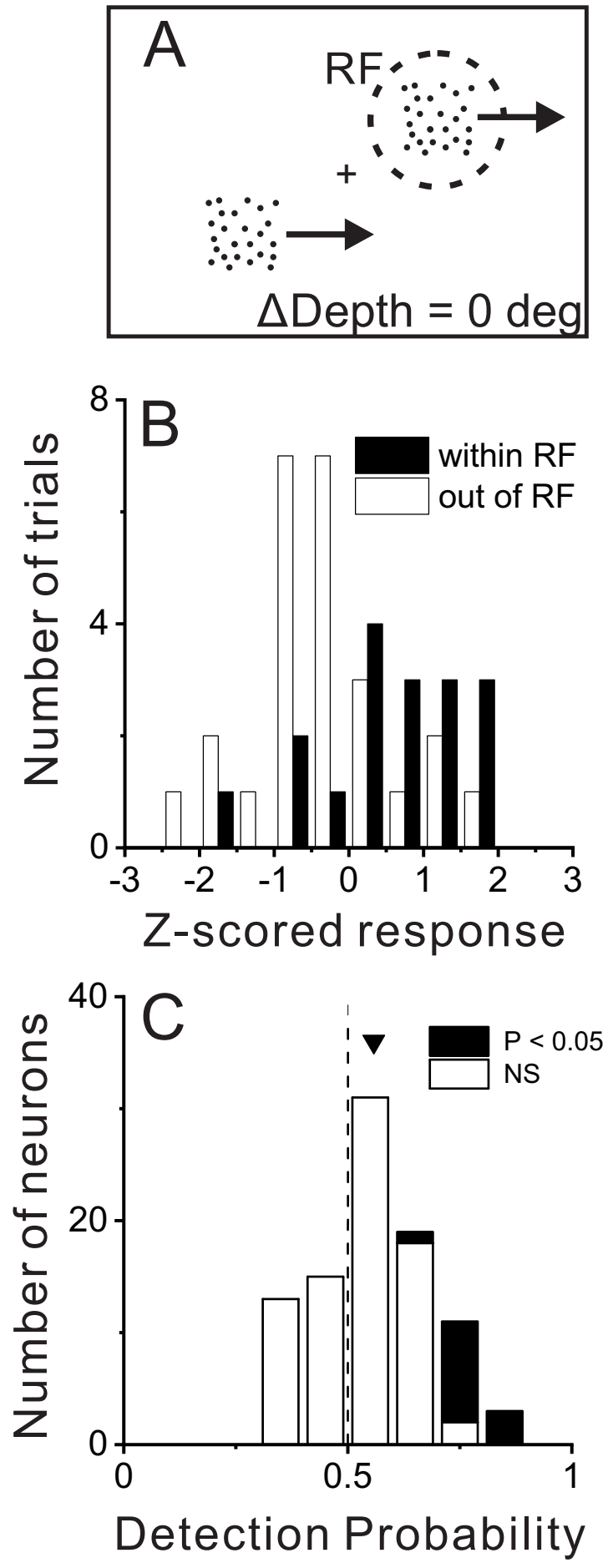


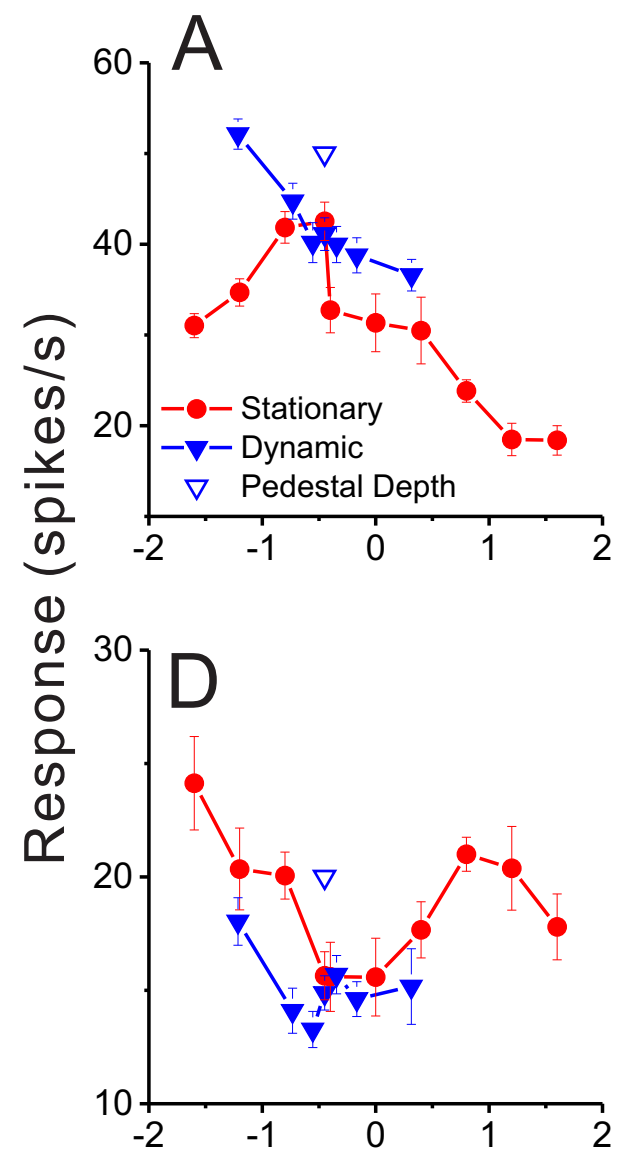

Depth from MP (deg)
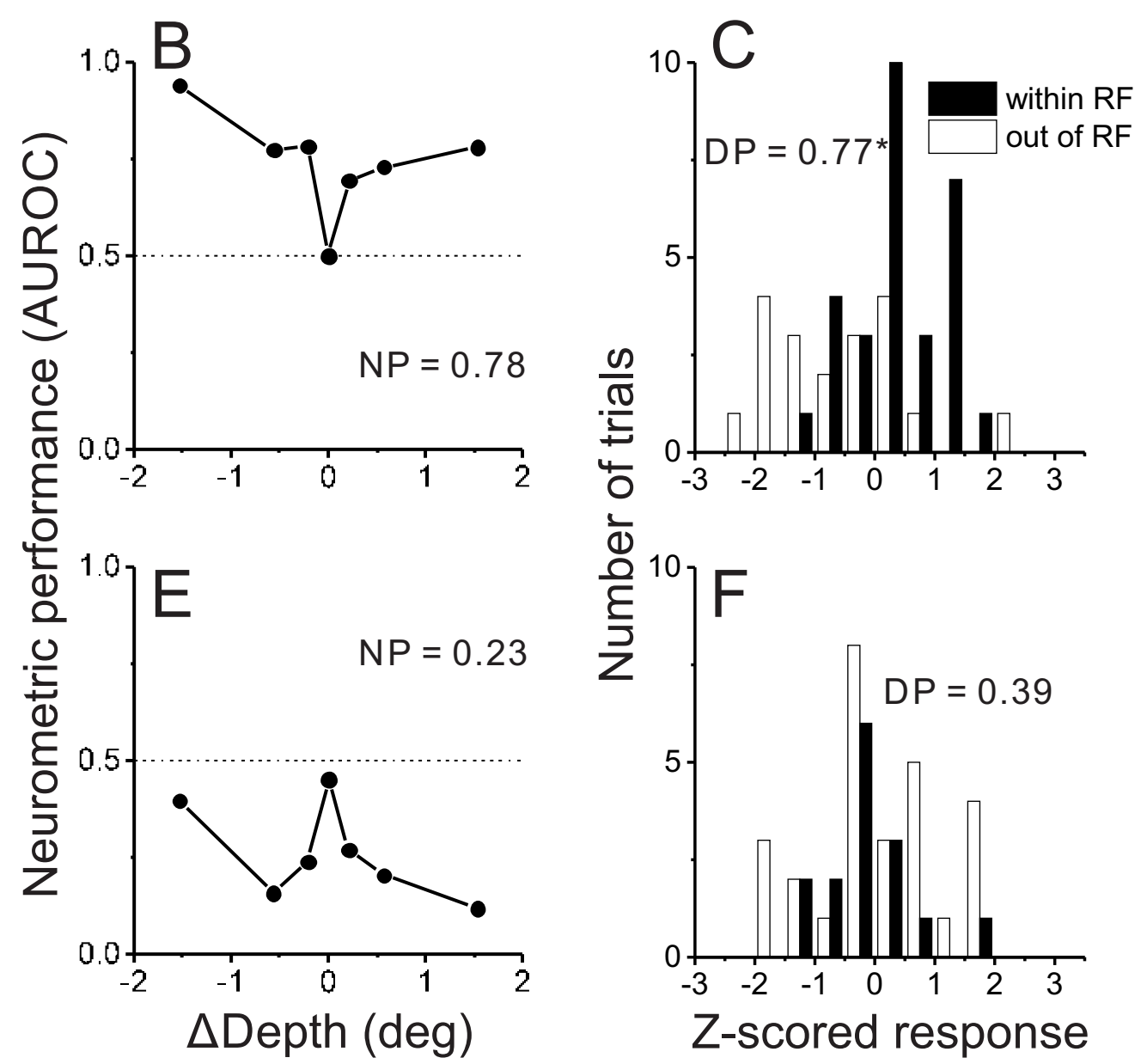

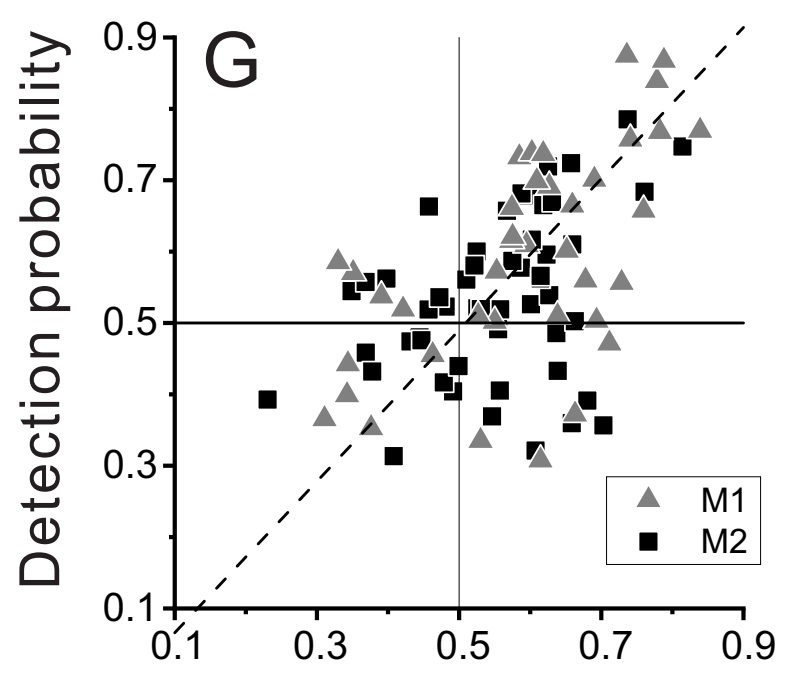

Neurometric Performance 
bioRxiv preprint doi: https://doi.org/10.1101/2021.11.16.468843; this version posted November 19, 2021. The copyright holder for this preprint (which was not certified by peer review) is the author/funder, who has granted bioRxiv a license to display the preprint in perpetuity. It is made available under aCC-BY 4.0 International license.
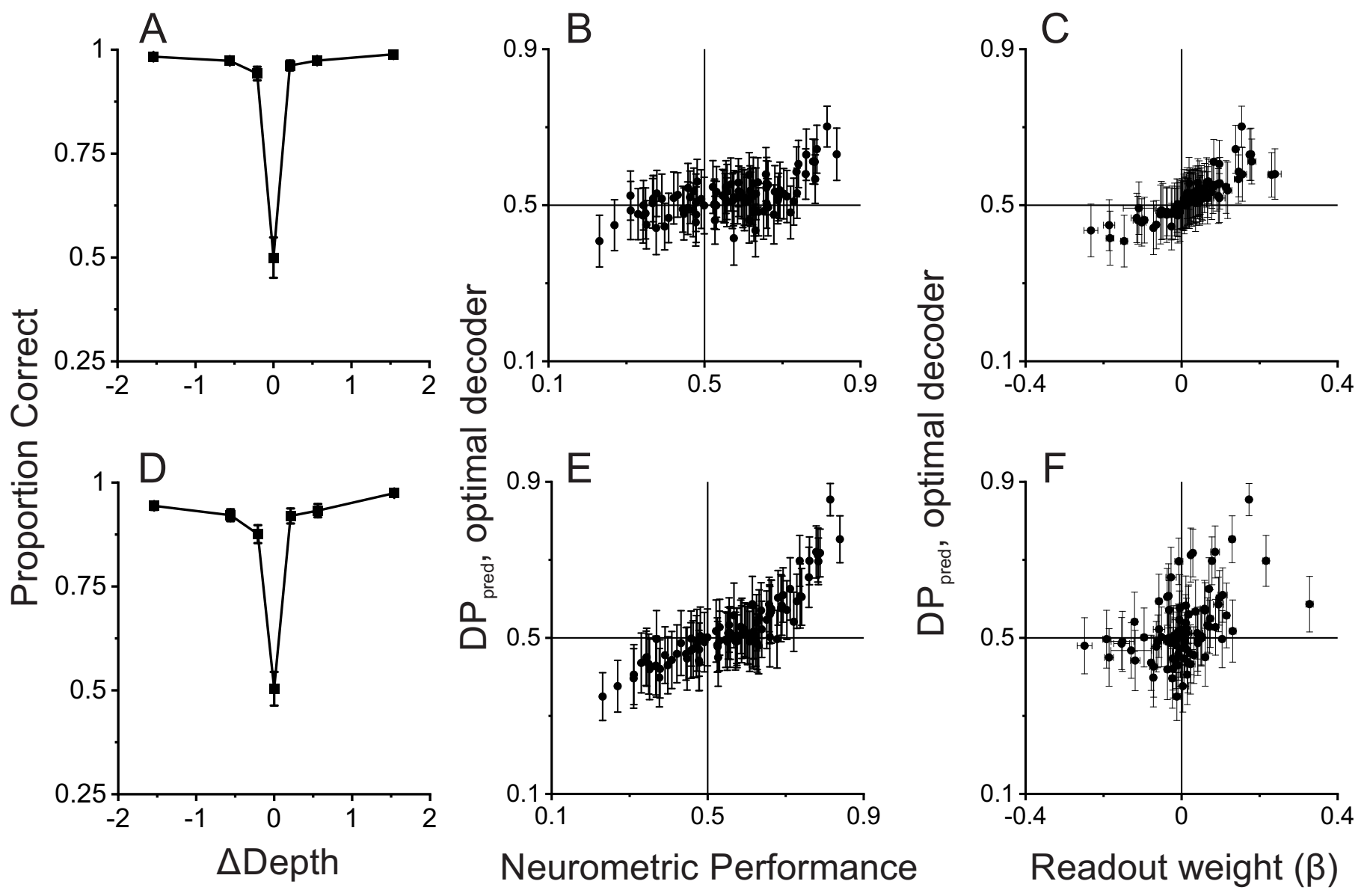
A
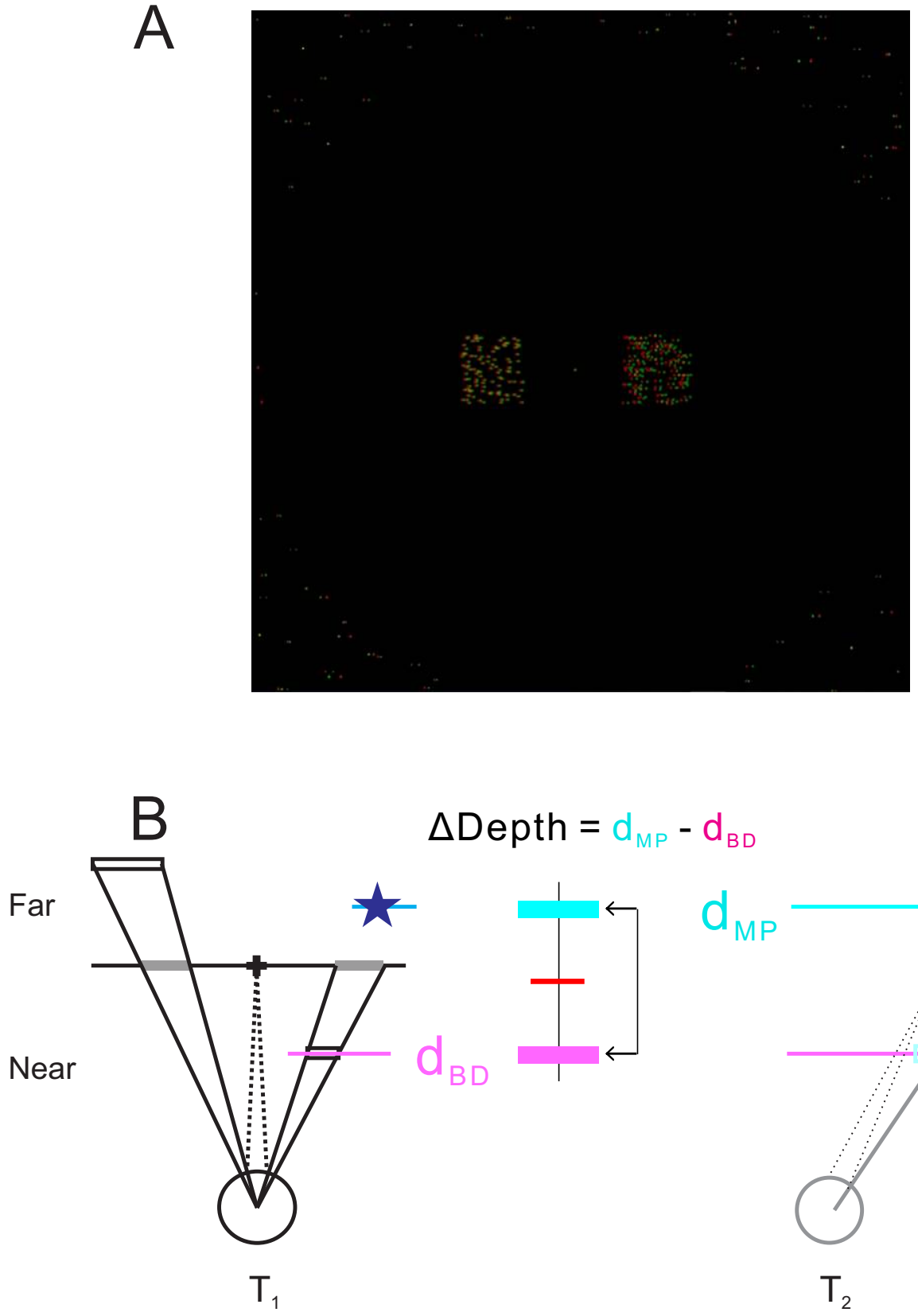

$-d_{B D}$
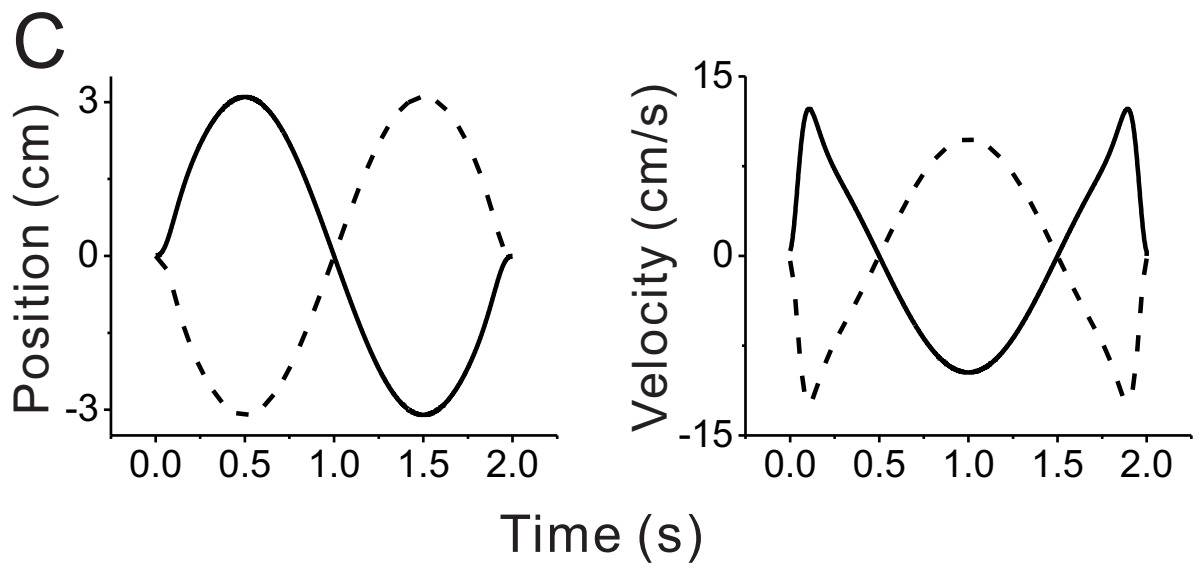

Supplementary Fig. 1 
bioRxiv preprint doi: https://doi.org/10.1101/2021.11.16.468843; this version posted November 19, 2021. The copyright holder for this preprint (which was not certified by peer review) is the author/funder, who has granted bioRxiv a license to display the preprint in perpetuity. It is made available under aCC-BY 4.0 International license.

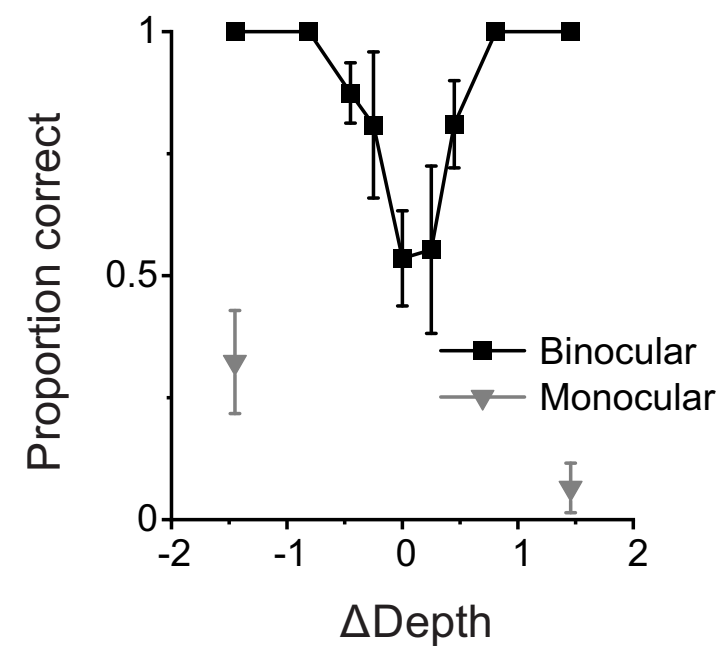



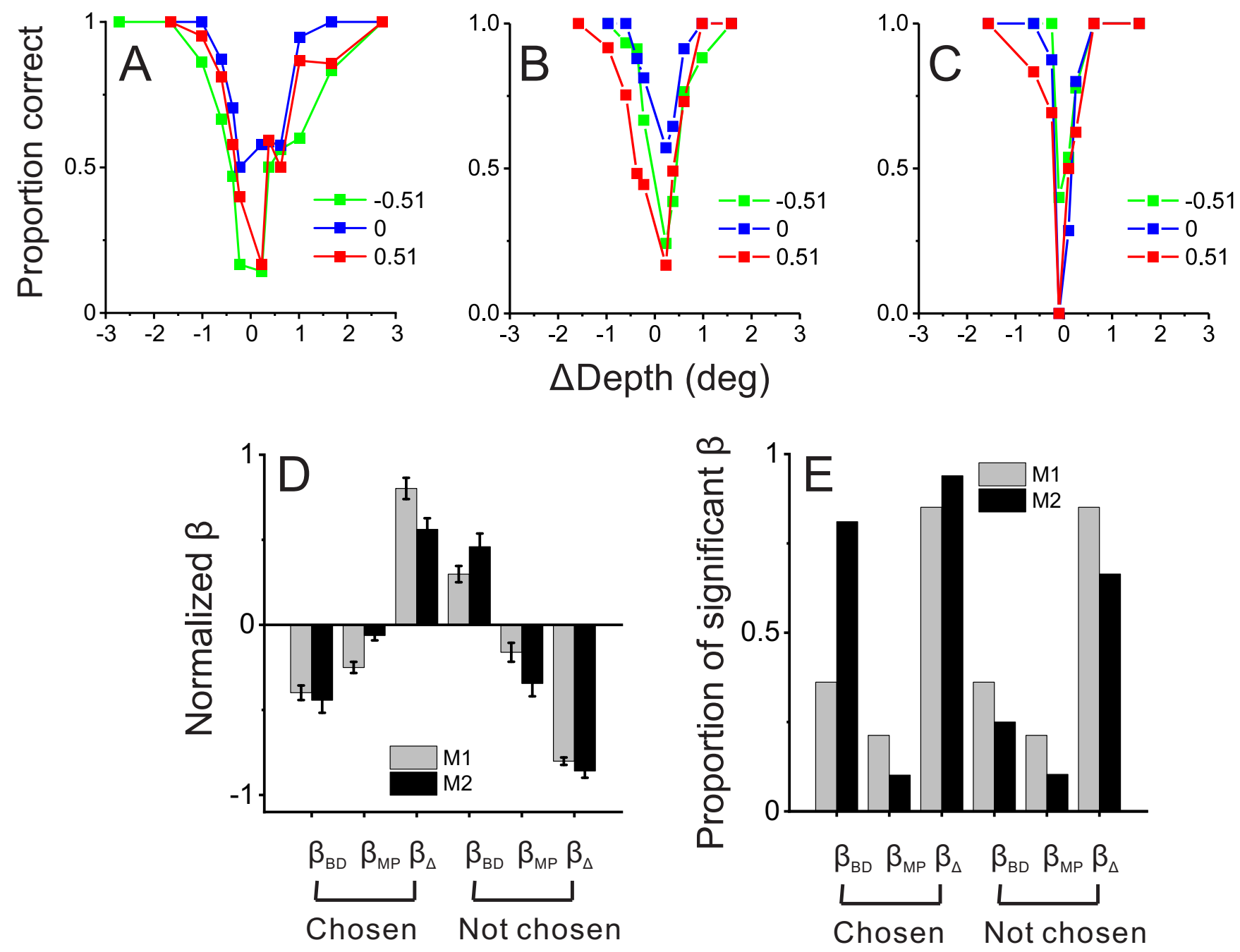
bioRxiv preprint doi: https://doi.org/10.1101/2021.11.16.468843; this version posted November 19, 2021. The copyright holder for this preprint (which was not certified by peer review) is the author/funder, who has granted bioRxiv a license to display the preprint in perpetuity. It is made available under aCC-BY 4.0 International license.
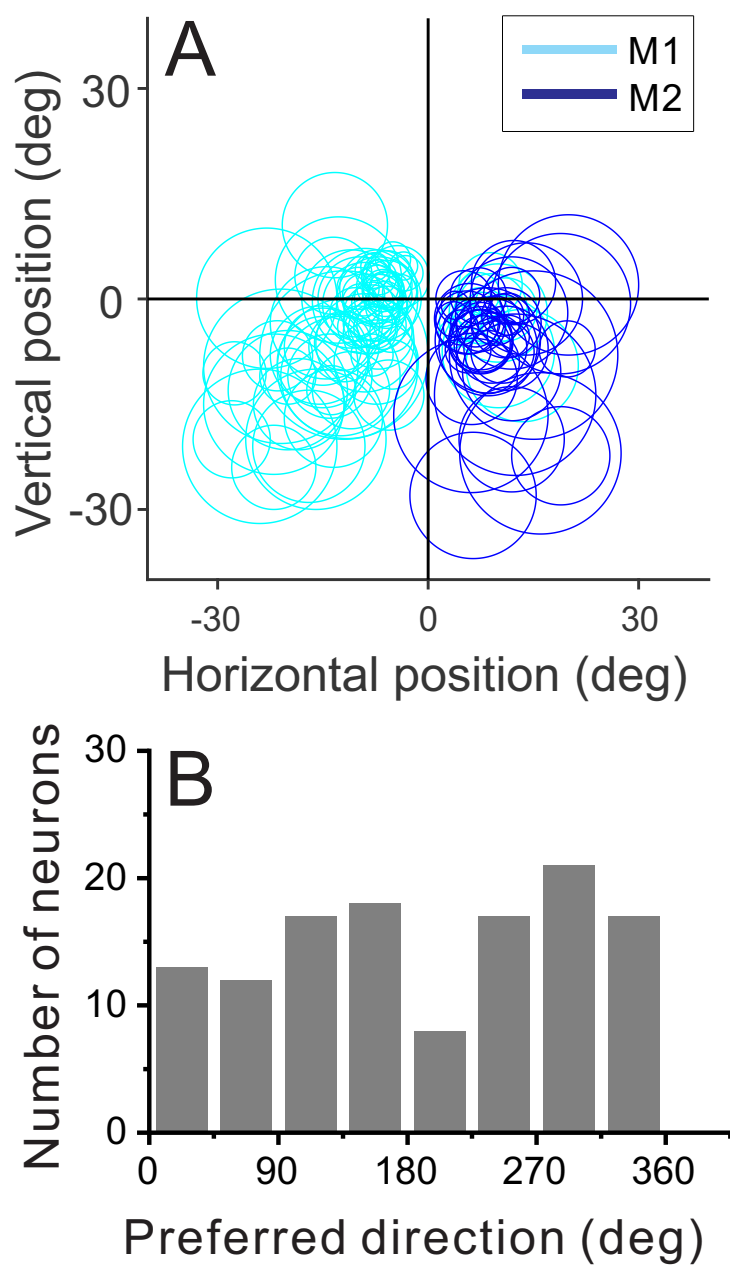

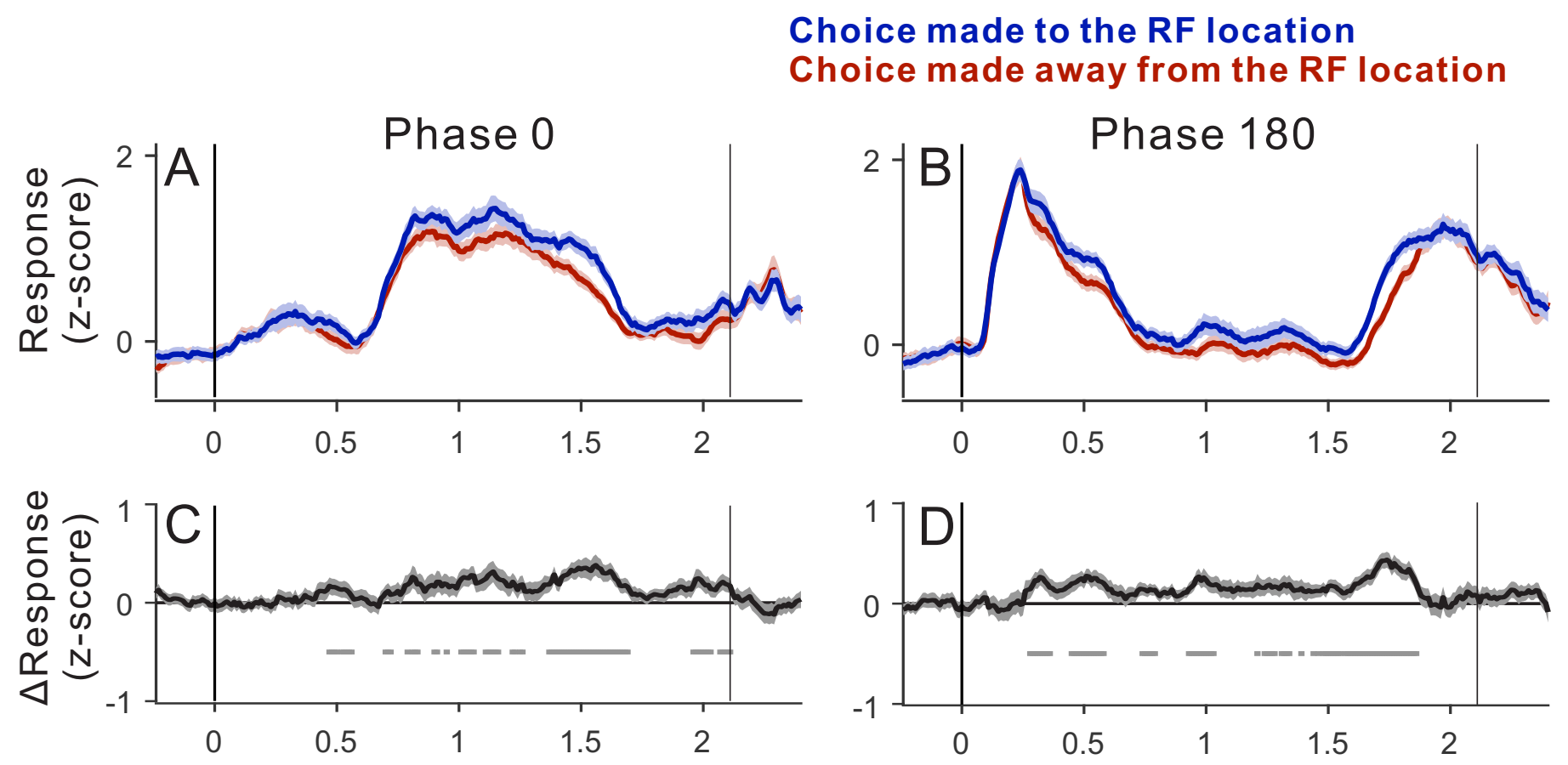

Time from self-motion onset (s) 